Discussion Paper No. 1138

\title{
Social Learning in Recurring Games
}

\author{
by \\ Matthew Jackson* \\ and \\ Ehud Kalai ${ }^{*}$
}

August 1995

*Department of Managerial Economics and Decision Sciences, J. L. Kellogg Graduate School of Management, Northwestern University, 2001 Sheridan Road, Evanston, Illinois 60208. e-mail: (Jackson) jacksonm@nwu-edu; (Kalai) e-kalai@nwu.edu. 



\title{
Social Learning in Recurring Games
}

\author{
loy
}

\section{Matthew Jackson and Ehud Kalai}

This Draft: July 1995

\author{
MEISS. Kellogg (iraduate Shool of Management. \\ Sorthwestern Eniversity. Evanston II. 6020x-2009
}

Abstract: In a recurring game. a stage game is played sequentially by different groups of players. Each group receives publicly available information about the play of carlier groups. Players begin with initial uncertainty about the distribution of types (representing the preferences and strategic behavior) of players in the population. Later groups of players are able to learn from the history of play of earlier groups. We first study the evolution of beliefs in this uncertain recurring setting and then study how the structure of uncertainty and information determine the eventual convergence of play. Wo show that beliefs converge over time and. moreover. that the limit beliefs are empirically correct: their forecast of future public information matches the true distribution of future public information. Next. we provide suffecient conditions to ensure that the play of any stage game is eventually close to that of a Bayesian equilibrium where players know the true type generating distribution. We go further to identify conditions under which play converges to the play of a tremblinghand perfect (Bayesian) equilibrium.

Wo are grateful to the ('alifornia Institute of Technology for their generous hospitality while we undertook this research, and thank the National Seienen Fonndation for financial support. Ehud Kalai also thanks the Sherman Fairchild Foundation. We thank Ehud Lehrer. Gilenn Loury and Roger Myerson for very helpful conversations. 



\section{Introduction}

Notions of repeated games are useful for modelling strategic dymamic interaction in economics. political. and of her social sciences. They also serve as experimental tools where convergenee properties of various fearning and evolutionary processes can be studied.

It is worthwhile to distinguish between two types of repetition. The first type is a repeated interaction among a fixed group of players. for example firms engaged in ongoing oligopolistic competition. Formal molels of repeated games describe such interartion and there is a large game theoretic litcrature in this area. (We refer the reader to Aumann and Hart (1992. 1994) for suvers and references.) A second type of repeated interaction. introduced formally as recurring games in this paper, involves different players at eacle time. As in a repeated game, in a recurring game a stage game is repeatedly played. but each stage is played by a new group of players. The repetition is important because each new group of players may observe relevant information about the play of arlier gronps. Recurring games capture the evolution of social behavior in multigenerational games, as well as interaction that occurs frequently in an existing society. For example. most of the real estate transactions which occur each year involve different participants and can naturally be modeind as a recurring game. Similarly, the numerous applications of a particular anction procedure at different times and locations constitute a recurring game. Not only are recurring games obviously applicalble to a wide variety of problems of practical interest. they also provide a wonderful laboratory for theories of learning and evolution.

Of particular interest here is the process of rational (Bayesian) learning in the context of recurring games. We study conditions under which play comes to approximate equilibrium play of the static stage games, and also identify interesting situations where such convergence fails. In addition. we explore how recurring game results about rational learning can be extended to include irrational players. long-lived players, and stochastio games.

Versions of reperaed and recurring games have been used to study fictitious play nave best response behavior. genetic and adaptive algorithms. Bavesian learning, and other learning and evolutionary systems. The import ant contributions to this literature are too numerous to list here. but examples related to our work here inchude: Sash (1950) and Aumann and Maschler (1967). as well as more recent work by Fudenberg and Kreps (1984). Jordan (1991). Fudenberg and Levine (1993). and halai and Leher (1993). Of this literature. 
the closest in spirit to the approach taken here are Jordan (1991) and Kalai and Lehrer (1993). ${ }^{1}$ Jordan (1991) studied a semi-rational repeated game Bavesian learning process that leads players" expectations in the long run to a Nash equilibrium. and Kalai and Lehrer (1993) studied a rational learning process that leads players in a repeated game in the long run to Nash equilibrium play. Our work is built on the same mathematical foundation of Bayesian npdating and convergence of beliefs. but differs substantially in the types of conclusions reached regarding convergence to various forms of equilibrium play. and the type of applications addressed.

To understand some of these differences. it is useful to note some overall differences betwen learning in recurring games and learning in repeated games. In a repeated game. for instance between a wife and a husband or a parent and a child. players have the time to learn to best respond to the actual players they face and their chosen strategies. On the other hand, in a recurring game. for example recurring single exchanges between pairs of buyers and sellers. such opportunities are not available. Thus. recurring game players who study the past plays of earlier players can at most learn the distribution of strategies in the population of opponents. but not the actual strategy of their realized opponents. So. in general, convergne in repeated games may lead to Nash equilibrium play while convergenee in recurring games may lead to Bayesian equilibrium play. This might be considered the "bad news."

There is atso some "good news" as one changes from repeated games to recurring ones. First. long run learning in a recurring game does not require that players live forever. as it does in a repeated game. Instead, only sufficient cumulative social experience must survive with time. This means that one does not have to be as conerned with the spend of convergence in recurring games. as one would in repeated games. Second. the imposition on players rationality is less severe in a recurring game. In a repeated game a player must solve an infinite horizon dyinamic optimization problem in addition to Bayesian learning.

1 Although the learning literature is closest in terms of the approach to modeling behavior. some evolutionary models are closer in terms of the fit into the recurring setting. For instance. Young (1993) studies the evolution of conventions in a recurring setting: a gane is played consecutively by different players and some observations of history are available to current players.

2 Of course. the news is bad only to the extent that one wants players to play Nash equilibrium. There are games for which players are better off in ignorance. i.e.. for which all Bayesian equilibrium outcomes Pareto dominate the Nash equilibrium outcomes. 
In a recurring game. the player's horizon can be very short. significantly simplifying the optimization problem. For instance, if the stage game requires only a single choice of action by a player, then there is no incentive to experiment and the behavior is simply myopic.

Whild the above myopic phenomenon makes the recurring analysis simpler. it can also create drastic welfare differences between the results of learning in recurring games versus learning in repeated games. Examples in this paper show that individual incentives not to experiment can lead to significant social welfare losses that may require social intervention. such as affirmative action. Experimentation by a player results in an externality relative to later players as it may help them leam. One can also find examples where the myopic behavior and failures to experiment lead to social gains.

Another phenomenon which is typical to recurring games is that there may be important differences in the information and monitoring between an outside observer. say the econometrician or the public. and the actual players of a stage game. Players from current or past stages of the game may know significantly more than outside observers who are only exposed to public signals from each stage. For this reason, the perceived social explanation for what is going on. may differ from the perspective of insiders which takes into account privately available information and experience concerning payoffs and actions which might not be publicly observed.

In arldition to the specialization to recurring games. the current paper also develops some general results and techniques regarding rational learning. First. by allowing a sifficiently rich set of beliefs in the minds of players. we obtain convergence to trembling hand perfect (Bayesian) equilibrimm. This contrasts with the previous fearning literature which obtains. at best. convergener to Nasle equitibrium. Second. a simple technique of representing players enables us to incorporate into the model players who do not learn at all. or who follow other methods of learning. One application of this technique is to examine the behavior of rational players in the presence of bonderly rational players. This is in the spirit of the analyis of Kreps. Milgrom. Roberts and Wilson (1952). A second application of this fechnigue shows that we can apply the recurring game results to situations that involve long-lived players. A third application allows us to adapt the model to capture situations where the game is stochastic. i.e.. changes stochastically from one stage to the next as a function of the social history. 
The paper is structured as follows. In the next section. three examples motivate and illustrate the approach and results. The following sections include the formal definitions for the general model and sufficient conditions for social learning to converge to Bayesian and trembling hand perfect Bayesian equilibrium play. Several additional examples illustrate various violations of the conditions and show how convergence may fail. The paper ends with a discussion of a method by which one can incorporate some long lived players and stochastic games into the model, and some conchuding remarks.

\section{Motivating and Illustrating Examples.}

Our first example shows a failing of learning in recurring games. We start with it because it highlights some of the differences between the recurring and repeated settings. In this example, social learning fails even with fully revealing signals because individuals do not have socially efficient incentives to experiment.

ExaMPte 1. Failed Social Learning: The Veed for Role Models and Affimative Action.

Consider the members of a population facing the option of taking on a certain task. e.g., attending law school. For the purpose of this example, divide the population into two types, those who would succeed at the task and those who would fail. An individual who strceeds receives a payoff of 1 , while an individual who undertakes the task and fails has a payofl of -2 . Not taking on the task results in a payofl of 0 for either type.

Without knowing his or her own type a member of the population decides whether to take on the task based on the type distribution in the population. For example. if the distribution of types in the population. $\tau$. is such that is of the population wonld succed. then the Bayesian equilibrium (optimal decision) relative to this prior $\tau$ is for every individual to take on the task $(.8 \times 1+.2 \times(-2)=.1>0)$.

If. however. members of the population are not sure about the distribution of types in the population, then less desirable social outcomes are possible. Suppose, for instance. that

3 This is easily fit into a Bayesian model as follows. Introduce a second player called "nature". whose type is either "succeed" or "fail". and who has no strategie role in the game (i.e. give player 2 only one action). The first player has only 1 type but his or her payoff depends on the realized type of player 2. This type of construction is described in detail in IIarsanyi (1967-68). 
members of the population believe that it is equally likely that the population is described by $\bar{\tau}$, which is just the reverse of $\tau$. That is, under $\bar{\tau}$. . $x$ of the population will fail. and the Bayesian equilibrium is to not take on the task.

Given the initial beliefs of the population that $\tau$ and $\bar{\tau}$ are equaly likely the first member of the population sees his or her chance of success as .5. and given the asymmetry in payoffs (i.e.. the higher cost of failure) chooses not to undertake the task. This means that the second member of the population to make this decision will have the same available information and will also choose not to undertake the task, and so forth. If $\tau$ is the true distribution, then since no one takes on the task. no one plays the optimal strategy relative to $\tau$. In this case. social learning fails. and inderel. this example violates the sufficient conditions for social learning that are presented in Section 6 .

While social policy is not the sub ject of this paper. notice that being aware of the failing of social Inarning abowe has policy implications. The social equilibrium has important informational externalities that are not taken advantage of. That is. if early individuals could be convinend to take on the task, against their own perceived individual incentives. with high probability their success rate will be observed by later individuals who will update the beliefs to put higher probability on the optimistic prior $\tau$ when it is the true distribution. In that case. after sufficiently long the optimal strategy will be for members of the population to take on the task.

This example illustrates a notion of positive affirmative action and role models. It would be to the benefit of the group as a whole even if they are not sure whether $\tau$ or $\bar{\tau}$ is the case. to induce early members of the population to undertake the task so that in the case $\tau$ "role models" will be created for the benefit of the large population to follow.

As a further remark on affirmative action, note that the incentives must be such that they do not obscure the success of the individuals. For example giving a population scholarships to law school. but not changing the bar exam requirements. will serve this purpose (unless the exam requirements are to be permanently changed). as changing the recuirements would produce less useful signals of the true distribution in the population.

The above example also illustrates clearly the difference between a recurring game and a repeated game. If taking on the task was a repeated action available to a single patient individual. then it would pay that individual to experiment in early stages. becanse of the 
potential benefit from learning his or her ability. Moreover, in the repeated game. the individual would learn about his or her own type, rather than the distribution of types in the population.

Example 2. Leaming to Coordinate in the Presene of Bonndedly Rational Players.

Consider a classic "Battle of the Sexes" game which is played recurringly. The payoffs are pictured below.

$\begin{array}{ccc} & A & \mathrm{~B} \\ \mathrm{~A} & 2.1 & 0.0 \\ \mathrm{~B} & 0.0 & 1.2\end{array}$

In addlition. consider that some players may be "boundedly rational" who follow a naive best response strategy, nbr for short. Such a player matches the last period action of the opposite sex player. For example. an nbr player in the role of player 1 at time $t+1$ adopts the action chosen by the player in the role of player 2 at time $t$. (Let first period nbr players randomize equally over $A$ and B.) Thus, an nbr player is implicitly assuming that the other player will choose the same thing that his or her predecessor did.

Assume that in each period. when a new pair of players is randomly drawn independently of each other and past players, they may be either Bayesian rational players or an nbr type. Each player knows only his or her own type and the social history of the actions chosen by both players in each stage preceeding their stage.

We begin by finding the Bayesian equilibrium strategies in the recurring game where the type generating distribution $\tau$ is publicly known to all players. With that in hand we will come back to the case where $\tau$ is nuknown. Suppose that under $\tau$ the probability of a player being an $n b r$ type is . $4(\tau(n b r)=.4)$, and of being a rational type is .6 $(\tau(r a t)=.6)$. The behavior of nbr players is easily predicted based on the play of the previous stage. We can thus compute the following Bayesian equilibrium strategies for the stage game at time $t+1$. where $t \geq 1$. by examining strategies of rational players and requiring that they be best responses to the distribution over the strategies of other rational players and $n b r$. players

Case 1. Actions were (A.A) at time $t$. 
The nbr types will play $A$ at time $t+1$ and this is also the unique equilibrium strategy for a rational player in either role. (A rational row player expects that the columm player will play i with probability at least .4 which makes i a best response for a rational row player. Then given that all row players will play $A$, this is the unique best response for a rational column player as well.) Thus the equilibrium actions in a stage following a stage of $(A . A)$ will be $(A . A)$ with probability 1 .

('ase 2. Actions were (B.B) at time $t$.

This is similar to case 1 and so the equilibrim actions in a stage following a stage of (B.B) will be (B.B) wilh probability 1.

(ase 3. Actions were (A.B) at time $t$.

In this case mbr types will play (B.A) at time $t+1$ in their respective roles. IIere the only equilibrium strategies for rational players are to play (A.B), in their respective roles. Rational players are swayed by the nbr types and play the opposite of them in an attempt to match their counterpart. (Matching the of her player if that player is an mbr type leads to the highest payoff. while matehing the other player if that player is rational leads to the lower payoff.) Thus, the equilibrium artions in a stage following a stage of (A.B) will be: (A.A) with probability.$(6 \times .4$. (B.B) with probability $.6 \times .4$. (A.B) with probability $.6 \times .6$. and (B.A) with probability .4X.4.

('ase 1. Actions were (B,A) at time t.

In this case nbr types will play (A.B) at time $t+1$ in their respective mles. For rational players. howeser, there are three sets of equilibrium strategies. Rational players could all play A with probability one in their respertive roles. Rational players could all play lis with probability one in their respective roles. Rational players could also all randomize to place probability $4 / 9$ on their respective favorite actions and $5 / 9$ on the of her action.

In the first equilibrinm. the actions in a stage following a stage of (B.A) will be: (A.A) with probability . $;$ and (A.B) with probability 4 .

In the second equilibrium. the actions in a stage following a stage of (B.A) will be: (B.B) with probability .6 and (A.B) with probability .4.

In the thied equilibrium. the actions in a stage following a stage of (B.A) will be: (A.A) 
with probability $2 / 9$, (B.B) with probability $2 / 9$, (A.B) with probability $4 / 9$. and (B.A) with probability $1 / 9$.

Notice that the cases of playing (A.A) and (B.B) (that is. ('ases 1 and 2 above) are absorbing when rational players follow their unique equilibrium strategies. Notice also that under recurring play. cases (A.B) and (B.A) must (with probability 1) eventually lead to one of these absorbing cases. We can thus conclude that in an equilibrium play of the above recurring game, where $\tau$ is known players will converge to coordinate forever on $(A . A)$ or (B.B).

Now let us consider what happens in the above situation if $\tau$ is not known to the players. Different equilibrium strategies may be realized. depending on the initial beliefs of the players concerning the relative likelihood of other type generating distributions. For example. players might believe that it is possible that an alternative $\bar{\tau}$ deseribes the relative likelihood of rational and nbr types. More generally. players may allow for other type generating distributions which assign probabilities to other types of irational players. For instance. they may believe that there are types of players who always play their favorite action. or players who best respond to what nbr's would do. ete. Clearly. there is a large set of type generating distributions of which the above $\tau$ is only one.

Our model captures this gane with uncertainty about the type generating distribution $\tau$ (the uncertain recurring game) as follows. First, a type generating dist ribut ion is randomly selected according to a commonly known prior distribution $\Gamma$. No one is told any information about the realized $\tau$. Next, the recurring game proceeds to be played. with player roles filled according to this unknown realized distribution $\tau$. As the game progresses. however. new players can upelate their beliefs about the realized $\tau$ based on the observed actions of players in rarlier stages.

Our theorem regarding comvergence to Bayesian equilibrium (Theorem 1. below) has strong implications here. Applied to this example it states that late players of the menertain recurring gane must platy close to the Bayesian equilibrium st rategies of their stage game. as if they knew the realized $\tau$. Moreover, additional conclusions of Theorem 1 are strong enough to preserve the the absorbing properties of the plays (A.A) and ( $B . B$ ). So even if the equilibrium players started with a highly diffuse prior over the distribution of ypes in the 
population. allowing for many types of boundedly rational behavior. they will learn to play as if they were playing a Bayesian equilibrium relative to the realized distribution. And. in particular. if the realized $\tau$ is the one we described earlier. then play will be absorbed to a recurring play of either $(A . A)$ or $(B . B)$.

We will return to a proof of the above conclusions (concerning absorption) after the formal presentation of the results.

As mentioned in the introduction. some of onr results provide conditions under which players will learn to play trembling hand perfect Bayesian equilibrium. In the previons examples there was no distinction betwern the Bayesian equilibria and the trembling hand perfect (Bayesian) equilibria of the static games. Thus, there was no room to distinguish between learning to play Nash equilibrium and learning to play a trembling hand perfect equilibrium. This distinction is made in the following example.

Example 3. Learning to ('oordinate on a Perfect Equilibrinm.

Consider a thee player game where payofs are known and given in the following table. where player 1 chooses a row. player 2 chooses a column, and player 3 chooses a matrix.

$\begin{array}{cccccc} & a_{3} & & & & \bar{a}_{3} \\ & a_{2} & \bar{a}_{2} & & a_{2} & \bar{a}_{2} \\ a_{1} & 1.1 .1 & 1.1 .1 & a_{1} & 3.3 .3 & 0.0 .0 \\ \bar{a}_{1} & 1.1 .1 & 1.1 .1 & \bar{a}_{1} & 0.0 .0 & 3.3 .3\end{array}$

In the right hand matrix players 1 and 2 play a coordination game. while in the left hand matrix the payoffs are constant. Player 3 can thus play $\bar{a}_{3}$ and hope that players 1 and 2 coordinate. or play $a_{3}$ and get a lower but sure payoff.

There are continua of Nash equilibria to the above game. There are 3 trembling hand perfect rquilibria of the above game: $\left(a_{1}, a_{2}, \bar{a}_{3}\right)\left(\bar{a}_{1}, \bar{a}_{2}, \bar{a}_{3}\right)$, and $\left(1 / 2,1 / 2, \bar{a}_{3}\right)$. where the $1 / 2$ indicates an reen mixing over the two available actions. Ail the trembling land perfect equilibria involve player 3 choosing $\bar{a}_{3}$. while there are Nash equilibria which involve player 3 playing $a_{3}$.

Consider a situation where all players are rational and they know the payoff table (and this is all common knowledge). In this situation, there are still a multitude of equilibria 
of the stage game from which to choose. The uncertainty that players face is thus strictly strategic. In this example, a player's type is simply an indication of what strategy they employ after each history. Players learn by observing the actions chosen in previous stages. For instance. all that matters to a first stage player 3 is his or her beliefs over the actions to be chosen by the other players. Thus, a type of the other player is what strategy they play. If player 3 has initial uncertainty which places high enough probability on coordination then 3 will choose $\bar{a}_{3}$. and otherwise 3 will choose $a_{3}$. Over time players will observe past plays of the game and learn to correctly predict the actions that will be chosen in subsequent stages. Thus, they will fearn to coordinate on an equilibrim. The question is whether that is simply a Nash equilibrium, or a trembling hand perfect equilibrium.

('onsider the strategies $\left(\bar{a}_{1}, a_{2} \cdot a_{3}\right)$. which constitute a Nash equilibrium that is not trembling hand perfect. We argue that if players have "full subjective uncertainty" in that they never completely rule out the possibility that the other platers will play any artion combination. and if players yypes are with low enough correlation. then they could only learn to play a trembling hand perfect equilibrium. Suppose to the contrary, that over time players have learned to coordinate on $\left(\bar{a}_{1}, a_{2}, a_{3}\right)$. Thuss. they play $\left(\bar{a}_{1} \cdot a_{2} \cdot a_{3}\right)$. but each player still has beliefs that place some probability on the event that the other players use any other combination of actions. Player 1 still believes that there is a positive probability (however small) that 3 will play $\bar{a}_{3}$. As long as player 1 does not believe that this action of player 3 is highly correlated with player 2 playing $\bar{a}_{2}$. then it is better for 1 to play $a_{1}$. rather than $\bar{a}_{1}$. This means that $\bar{a}_{1}$ could not be a best response to player 1 s beliefs and so $\left(\bar{a}_{1} \cdot a_{2}, a_{3}\right)$ conld not be the result of rational learning.

It is clear that the role of the correlation in the beliefs of plaver 1 conerning the possible actions of players 2 and 3 is important in the above example. Civen full subjective uncertainty this correlation determines whether convergence ends up being to a trembling hand perfect equilibrium. or to an undominated Bavesian equilibrim which might not be trembling hand perfect. This is explored in more detail in Example 7 . Theorem 3. and the concluding remarks.

We furn now to a formal presentation of the model and results. 


\section{Recurring Games}

In a recurring (Bayesian) game. a stage game is played at each time $t \in\{1.2 .3 \ldots\}$. New players are randomly drawn at each time $t$ to play the stage game.

The stage game is a standard Bayesian game described by a list $\left(X,\left\{A_{i}, \Theta_{i}, u_{i}\right\}_{i \in N}, \tau\right)$ with the following interpretations. The set $Y=\{1,2 \ldots n\}$ describes player roles with $A_{i}$ describing a finite set of (pure) actions available to a player in role $i . A=A_{1} \times \cdots \times A_{n}$ describes feasible action combinations. The notation $\Delta\left(A_{i}\right)$ denotes the mixed actions available to a player in role $i$. Each $\Theta_{i}$ is a countable set describing the possible types of a player in role $i$, and $\Theta=\Theta_{1} \times \cdots \times \Theta_{n}$ describes profiles of players types. $\tau$ is a type generating distribution, which is a probability distribution defined orer the profiles of types in $\Theta$. Players payofs depend both on the vector of types and the chosen actions. Player $i$ s preferences are represented by the von Neumann- Morgenstem ntility function $u_{i}: A \times \Theta-M R$. Etilities are bounded: there exists a finite $M \geq 0$ such that $\left|u_{i}(a, \theta)\right| \leq M$ for all a and $\theta$.

To describe the recurring game. it is necessary to define the way in which information becomes available from each stage. $S$ is the countable set of possible publicly obsersed signals, with generic element $s \in S$. Each vector of stage game artions a $\in A$ and type $\theta \in \Theta$ results in signals according to a probability distribution $\mu_{n, \forall}$ defined over $S$. A (social) history of length $t$, denoted $h^{t}$, is a vector of publicly observed signals $\left(s^{1}, s^{2} \ldots \ldots s^{t}\right) \in S^{t}$. Players at time $t=1$ will not have observed any signals, so we adopt the convention that $s^{0}=\{\emptyset\}$. Let $I I=s^{0} \cup\left(\cup_{t} S^{t}\right)$ be the set of all possible social histories.

A player in the renuring Bayesian game is denoted (i.t). representing the player of role $i$ at time $t$. The recurring Bayesian game is thus described as follows.

Initially (after history $\left(\right.$ ), a vector of types $\theta^{1}$ is randomly drawn according to $\tau$. Each player (i. $)$ is informed of lis or her respective $\theta_{i}^{1}$. Next. each player (i. 1$)$ chooses an action $a_{i}^{1} \in A_{i}$. Players are paid $u_{i}\left(a^{1}, \theta^{1}\right)$ and a signal $s^{1}$ is randomly drawn aconding to $\mu_{a^{1}}, \theta^{1}$. $h^{1}=\left(s^{1}\right)$ becomes the publicly known social history (of length 1$)$. In the second stage. a new vector of types. $\theta^{2}$. is is randomly drawn according to $\tau$ and ach new player (i.2) is informed of his or her respective type $\theta_{i}^{2}$ and chooses an action $a_{i}^{2} \in A_{i}$. Players are paid $u_{i}\left(a^{2}, \theta^{2}\right)$ and a signal $s^{2}$ is randomly drawn according to $\mu_{1^{2}, y^{2}}$. The next social history is $h^{2}=\left(s^{1}, s^{2}\right)$. The recurring game is defined inductively in the manner just described. 
Strategies for players in a recurring gaue are represented by maps $\sigma_{i}: I I \times \Theta_{i} \rightarrow \Delta\left(A_{i}\right)$. which prescribe a mixture over the possible pure actions available to each player role as a function of the realized type in that player role and the observed history up to that stage. The utility of a player in the recurring game for a given profile of strategies is defined to be the expected payof in the obvious way.

\section{Uncertain Recurring Games}

In a recurring game. if the players know the distribution according to which types are drawn $(\tau)$. then the analysis is similar to the analysis of a static Bayesian game las developed by Harsanyi (1967. 1968)). It differs in that the history of previous stages may serve as a correlating deviee. If the players do not know the distribution $\tau$. then history also plays an interesting role in learning. This is the focus of onr analysis and is captured as follows.

Let $M(\Theta)$ be a set of probability distributions over $\Theta$. The nnertainty of players is represented by $\mathrm{r}$. a probability distribution over $M(\Theta)$. We assume that $\Gamma$ has countable support, and thus also assume that $M(\Theta)$ is countable. Prior to the start of the recurring game. a type generating distribution $\tau \in M(\Theta)$ is drawn according to the known distribution I. The players then proceed to play the recurring game without any information about the realized $\tau$. However, since players in each stage know l' and see the history of previons play. over the course of the uncertain recurring game. they update $T$ according to the observed history of public signals and their own type.

Remark: It is important that we have modeled the uncertainty in the form of a prior over priors. The game is "doubly Bayesian" in the sense that players of any stage are unsure as to which Bayesian game they are playing. They learn about which Bayesian game they are playing from updating based on the social history. If one tried instead to model this by simply endowing players with a possibly incorrect prior over types. then there would be no way for players to learn from the social history.

Remark: We require that all players start with the same uncertainty l. This consistency assumption is common in the literature on Bayesian games. Nevertheless, this assumption

4 Sotice the comvenient abuse of notation. A full description has $\sigma$ as a function of $i . t$. since each pair $(i, t)$ is a new player. Howewer. since $t$ is identifiable from a history, we economize on the notation. 
is restrictive to the degree that heterogeneity in beliefs over different $\tau$ 's conditional on histories cannot be incorporated into the type space. As will become obvious, the basic learning and convergence to equilibrium results are not dependent on the common prior. and extend easily to the case of diverse priors. The modifications which are necessary concern the fact that if there are an infinite number of different priors then there might be no uniform rate of convergence."

\section{The Probability Space}

Before proceeding. it will be helpful to define a probability space which serves as the basis for an uncertain recurring game. given a strategy $\sigma$.

A fully described ontcome (or state) is an infinite sequence $\left(\tau, \theta^{1}, a^{1}, s^{1} \cdot \theta^{2} \cdot a^{2} \cdot s^{2} \ldots\right)$ in $M(\theta) \times(\Theta \times A \times S)^{x}$. To describe the probability distribution over outemes, it suffices to define consistent probabilities over all initial seguents of ontcomes. and then $P_{\sigma}^{\prime}$ is the consistent extension to the set of all outcomes. ${ }^{6}$ This is done inductively by letting $P_{\sigma}(\tau)=\Gamma(\tau)$ and

$$
P_{\sigma}\left(\tau \ldots \ldots s^{t} \cdot \theta^{t+1} \cdot a^{t+1} . s^{t+1}\right)=P\left(\tau \ldots \ldots s^{t}\right) \tau\left(\theta^{t+1}\right) \sigma_{\theta^{t+1}, k^{t}}\left(a^{t+1}\right), \mu_{n^{t+1}, \theta^{t+1}}\left(s^{t+1}\right) .
$$

where $h^{t}=\left(s^{1} \ldots s^{t}\right)$.

The updating that players undertake is captured by $P_{\sigma}\left(\tau \mid h^{t} \cdot \theta_{i}^{t+1}\right)$, a version of the conditional probability distribution.

\section{Social Learning}

The first lemma states that an observer who updates the prior distribution $\Gamma$ according to the observed histories will eventualy stop learning, in the sense that the updated I $\left(P_{\sigma}^{\prime}\left(\tau \mid h^{t}\right)\right)$ converges to a limit distribution. The convergence of the probability placed on any $\tau$ is a standard conseguence of the martingale convergence theorem. The fact that the

5 We would also need to add an absolute continuty condition, requiring that each player"s beliefs $\Gamma_{\theta}$ be absolutely continuous with respect to the true distribution $\Gamma$. In the absence of this condition. a player might a priori rule out the realized distribution $\tau$ and would then be nuable to learn.

6 We abuse notation and let $\tau$ denote the erent consisting of all outcomes with first entry $\tau$. and $\tau \ldots \ldots \theta^{t} \cdot a^{t}, s^{t}$ denotes the event consisting of all outcomes with this initial segment. etc.. To complete the probability space, consider the $\sigma$-field generated by the set of events which consists of all finite initial segments. 
resulting limits together still constitute a probability distribution requires a bit more work. which is given in the proof.

Lemma 1: Converciexce of Beliefs. Consider an uncertain recurring game and a list of strategies $\sigma$. For almost every $h \in S^{\infty}$ there exists $\Gamma^{x} \in M(\Theta)$ such that $I_{\sigma}\left(\cdot \mid h^{t}\right)$ converges to $\Gamma^{x}$.

ProOf: Consider any $\tau \in M(\Theta)$. Let $X^{t}=P_{\sigma}\left(\tau \mid h^{t}\right)$. The sequence of $X^{t}$ s sis a martingale. By the martingale convergence theorem (for a statement see Theorem 35.4 in Billingsley $(1979))$, there exists a random variable $X$ such that $X^{t}$ converges to $X$ almost surely. For each $\tau$ define $\Gamma^{x}(\tau)$ to be this $X$. Let $\Omega_{\tau}$ be the outcome set (of measure 0 ) for which this convergence fails. Since $M(\Theta)$ is countable, it follows that $U_{\tau} \Omega_{\tau}$ is a set of measure zero.

We now show that $\sum_{\tau \in M(-))} \Gamma^{\alpha x}(\tau)=1$, almost surely. This implies that $\Gamma \in M(\Theta)$ (almost surely), and that $P\left(\tau \mid h^{t}\right)$ converges to $\Gamma^{x}(\tau)$ uniformly across $\tau$ (almost surely). This is accomplished in two steps. First. we will show that

$$
\sum_{\tau \in M(-))} \Gamma^{\infty}(\tau) \leq 1
$$

for every outcome outside of $U_{\tau} \Omega_{\tau}$. Next. We will show that

$$
E\left[\sum_{\tau \in M(\epsilon))} \Gamma^{x}(\tau)\right]=1 .
$$

Together. (1) and (2) imply that $\sum_{\tau \in M(\Theta)} I^{x}(\tau)=1$. almost surely.

To prove (1). suppose to the contrary that $\sum_{\tau} I^{\prime x}(\tau)>1$ for some outcome outside of $U_{\tau} \Omega_{\tau}$. Let $h$ be the history of signals associated with this outcome. Then there exists a finite $B \subset M(\theta)$ such that $\sum_{\tau \in B} \Gamma^{x}(\tau)-1=d>0$, for this outcome. Let $n=$ \#B. Since $B$ is finite. there exists $T$ such that $\mid P_{\sigma}\left(\tau \mid h^{t}\right)-\left[{ }^{\infty}(\tau) \mid<d / n\right.$ for all $\tau \in B$ and $t \geq T$. This implies that $\sum_{\tau \in B} I_{\tau}^{\prime}\left(\tau \mid h^{t}\right)>1$ for any $1 \geq T$. a contradiction.

To prove (2). fix any finite $B \subset M(\theta)$. First we show that

$$
E\left[\sum_{\tau \in B} \Gamma^{\alpha^{*}}(\tau)\right]=\sum_{\tau \in B} \Gamma(\tau) .
$$

Since $B$ is finite it follows that $E\left[\sum_{\tau \in B} \Gamma^{x}(\tau)\right]=\sum_{\tau \in B} E\left[\Gamma^{\infty}(\tau)\right]$. Which by definition is equal to $\sum_{\tau \in B} E\left[\lim _{t \rightarrow x} P_{\sigma}\left(\tau \mid h^{t}\right)\right]$. By Lebesgue ${ }^{\prime}$ bounded convergence theorem (for a

${ }^{7} P_{\sigma}\left(\cdot \mid h^{t}\right)$ converges to $\Gamma^{x}$ at $h$ if for any $\epsilon$ there exists $T$ such that for all $t>T$ $\sup _{\tau \in M(t)}\left|I_{\sigma}^{\prime}\left(\tau \mid h^{t}\right)-\Gamma^{\infty}(\tau)\right|<\ell$. 
statement see Billingsley (1979). Theorem 16.4), it follows that

$$
\sum_{\tau \in B} E\left[\lim _{t \rightarrow \infty} I_{\sigma}^{\prime}\left(\tau \mid h^{t}\right)\right]=\sum_{\tau \in B} \lim _{t \rightarrow \infty} E\left[P_{\sigma}\left(\tau \mid h^{t}\right)\right]
$$

Since, $E\left[I_{\tau}\left(\tau \mid h^{t}\right)\right]=\Gamma(\tau)$ for all $t$, we have established (3). One easily establishes (2) using (3) and the fart that $\sum_{\tau \in M(\Theta)} \Gamma(\tau)=1$.

Athough Lemma 1 establishes that an observer's beliefs will converge over time. it does not guaranter that they converge to the true distribution $\tau$. or even that the observer will be making correct predictions conrerning forthcoming public signals. This second conclusion, however, intuitively follows from the convergence of baliefs. If the predictions are still significantly incorrect at some time, then there is room for additional learning. Thus. once learning has effectively stopped, predictions should be approximately correct. This is captured in Lemma 2. below.

First. we define beliefs to be e-mpirically correct if they induce a distribution over signals which is e close to the actual distribution over signals.

Definition: Beliefs are empirically correct relative to $\tau$ and $h$ after $T$ if $\Gamma_{\sigma}^{\prime}\left(s^{t+1} \mid h^{t}\right)$ is c $\operatorname{close}^{x}$ to $P_{\tau}\left(s^{t+1} \mid \tau \cdot h^{t}\right)$ for all $t \geq T$.

An empirically correct set of beliefs are those where an observer of social histories, $h^{t}$. has learned to predict signals as if he or she knew the realized distribution $\tau$.

The following result can be proven directly from Theorem 3 in Kalai and Lehrer (1993a). (See also Blackwell and Dubins (1962).)

Lemma 2: Socin I. Farsixg. For any list of strategies $\sigma$. distribution $\tau$. and $\epsilon>0$. there exists a random time $T$ such that the (Bayesian updated) beliefs are e-empirically correct after $T$.

Lemma 2 fells us that over time. based on the history of the recurring game. Bayesian observers who start with uncertainty $\Gamma$ will learn to predict the signal that will occur in any stage as if they knew the true distribution. $\tau .{ }^{10}$ Lemma 2 does not imply that players

$8 \mu^{\prime}$ is $6-c$ lose to $\mu$ if there exists a measurable set $Q$ such that (i) $\mu(Q)>1-\epsilon$ and $\mu^{\prime}(Q)>1-\epsilon$ and (ii) $(1-c) \mu^{\prime}(A) \leq \mu(A) \leq(1+\epsilon) \mu^{\prime}(A)$ for every measurable $A \subseteq Q$.

\$A random time means that for almost every outcome there exists such a $T$.

10 In fact. the stronger conclusion that they will correctly predict all events involving future signals is true. In a recurring game. however. players only care about their own stagr. 
will come to know $\tau$. or that they will correctly predict signals conditional also on their type (see Example 5). We now turn to an examination of these issues as they pertain to the convergence of equilibrium play in uncertain recurring games.

\section{Equilibrium in Uncertain Recurring Games}

Given an uncertain recurring game. let us define the expected utility of player $i$ for a given profile of strategies $\sigma$. conditional on a history of signals through time $t . h^{t}$. and player is type $\theta_{i}^{t+1}$ :

$$
\bigvee_{i}\left(\sigma, h^{t} \cdot \theta_{i}^{t+1}\right)=\sum_{\theta^{t+1} \in \Theta, i^{t+1} \in A} P_{\sigma}\left(a^{t+1} \cdot \theta^{t+1} \mid h^{t} \cdot \theta_{i}^{t+1}\right) u_{i}\left(a^{t+1} \cdot \theta^{t+1}\right) .
$$

A profile of strategies $\sigma$ forms an uncertain Bayesian equilibrium of the uncertain recurring game if. for all $i . l . h^{t} \in S^{t}$. and $\theta_{i} \in \Theta_{i}$.

$$
V_{i}\left(\sigma, h^{t} \cdot \theta_{i}\right) \geq V_{i}\left(\sigma / \tilde{a}_{h^{t}, \theta_{1}} \cdot h^{t}, \theta_{i}\right)
$$

for all $\widetilde{a}_{h^{t}, \psi_{1}} \in A_{i}$. where $\sigma / \widetilde{a}_{h^{2}, \theta_{1}}$ is the profile of strategies which alters $\sigma$ only by changing $\sigma_{i}$ to $\tilde{a}_{h^{2}, A_{1}}$ after history $h^{t}$ for type $\theta_{i}$.

\section{Convergence to Bayesian Equilibrium.}

As illustrated in our previous examples. the consequences of learning depend on the informativeness of signals and the structure of the uncertainty. In this section. we present conditions under which social learning leads players in the uncertain recurring game to eventually play actions which approximate a Bayesian equilibrium relative to the true (realized) type generating distribution. We then provide examples that illustrate the roles of these conditions by showing what can happen if these conditions are violated.

In the remaining sections. we restrict attention to situations where signals are deterministic: for each $a$ and $\theta$ there exists ss such that $\mu_{a, \theta}(s)=1$. The notation $s_{n, \theta}$ replaces the distribution $\mu_{t, \theta}(s)$. Sotice that this does not imply that signals are fully revealing. as different action-type pairs could map into the same signal.

Definition: A recurring game has payoff sufficient signals if. for all $i$ and $a, \theta, \bar{a}, \bar{\theta}$ such that $\bar{\theta}_{i}=\theta_{i}$ and $s_{u_{1}, \dot{H}}=s_{\bar{u}, \bar{\theta}}$. it follows that $u_{i}\left(\widehat{a}_{i}, a_{-i}, \theta\right)=u_{i}\left(\hat{a}_{i}, \bar{a}_{-i}, \bar{\theta}\right)$ for all $\hat{a}_{i}$. 
The condition of payoff sufficint signals states that the information contained in a signal. when coupled with the information of a player's type is sulficient for that player to calculate his or her anticipated payoff as his or her action varies.

This condition is (when coupled with the next condition) sufficient for play of an uncertain Bayesian equilibrium to converge to that of a static Bayesian equilibrium relative to the true distribution. The intuition is straightforward. Players eventually learn the correct distribution over signals. If this information. together with the rest of their information (their type) allows them to correctly calculate their payoffs, then they are choosing a best response to the distribution over their payoffs indued by the strategies of the other players under the true distribution. In the absence of this condition. players are missing payoff relevant information and coukl choose strategies which are best responses given the information that they have but not to the true underlying distribution.

The payoff suflicient signals is ahmost a necessary condition for play to converge to resemble the play of a Bayesian equilibrium where playes know the type generating distribution. It is not quite necessary. because cren in the absener of any information it is possible that best responses to the initial uncertainty happen to be the same as the best responses where players know the realized type generating distribution.

Let 11 mention two situations where it is clear that there are payoff sufficient signals. First. if players payoffs are private valued and signals are the actions played in a stage then signals are payoff suflicient. Second, if there are common values and signals reveal types and actions then signals are payoff sufficient.

Having payolf sufficient signals alone is not quite enough to guaranter convergence to a Bayesian equilibrium relative to the true (realized) distribution. since it is possible that one has learned to correctly predict signals unconditionally. but has not learued to correctly predict them given the additional information contained in one"s own type. (This is illustrated in Example (j.) It is ruled out by the following condition.

Definition: Social lraming implies priate learning relative to $\sigma$ if for any $\delta>0$ there exists $\epsilon>0$ such that for any $h \in S^{x}$ if $P_{\sigma}\left(s^{t+1} \mid h^{t}\right)$ is $\epsilon$ close to $P_{\sigma}\left(s^{t+1} \mid \tau \cdot h^{t}\right)$ for all $t$ above some $T$. then $P_{\sigma}\left(s^{t+1} \mid h^{t}, \theta_{i}^{t}\right)$ is $\delta$-close $P_{\sigma}^{\prime}\left(s^{t+1} \mid \tau, h^{t}, \theta_{i}^{t}\right)$ for all $t>T$ and any $i$ and $\theta^{t}$ such that $\tau\left(\theta^{t}\right)>0$. 
The social learning implies private learning condition, is easily interpreted. It states that whenever an outside observer has learned to approximately predict the signals of the next stage as if le or she knew the true type generating distribution. then any inside observer (who gets an additional piece of information $\theta_{i}$ ) will also be able to approximately predict the signals of the next stage as if he or she knew the true type generating distribution.

We mention two situations where it is clear that sorial learning implies private learning. (i) Each distribution $\tau$ in the support of $I$. coupled with the strategies $\sigma$ learls to a different distribution over signals. In such a case any observer who learns the distribution over signals will also learu the true type generating distribution. (ii) Types are drawn independently so that each $\tau$ is a product of $\tau_{i}$ "s and l' can be decomposed into a product of distributions across $\tau_{i}{ }^{\circ}$, and either signals are the actions played, or signals are the types drawn, or the signals are the actions played and the types drawn. Here one's own type does not tell one anything new about the types or actions of the other players.

Situations where social learning does not imply private learning require particular interdependencies among types, as illustrated in Example 6 .

Before we state lheorem 1 . it is necessary to define ideas of closeness to a Bayesian equilibrium relative to a given distribution. There are two conclusions to the theorem. First. that after a sufficient time players choose artions yielding $\epsilon$ optimal expected utilities as captured in the definition of a tight $\epsilon$-Bavesian equilibrium, below. Second, after a sufficient time players" strategies play close to an actual Bayesian equilibrium of the true static game. This closeness is formalized in the defuntion of plays c-like.

Definition: A profile of strategies, $\sigma$. form a tight $\epsilon$. Bayesian equilibrimm of static Bayesian game (...A.,$\left.. \tau .\left\{u_{i}\right\}\right)$ if for all $i, \theta_{i} \in \Theta_{i}$. and $a_{i}$ in the support of $\sigma_{i}\left(\theta_{i}\right)$

$$
V_{i}\left(\sigma / a_{i}, \tau, \theta_{i}\right) \geq V_{i}\left(\sigma / \bar{a}_{i}, \tau \cdot \theta_{i}\right)-\epsilon
$$

for all $\bar{a}_{i} \in A_{i}{ }^{11}$

Notice that a tight e-equilibrium is stronger than a standard definition of e-equilibrium (as in Radner (1980)). The strenghening is that every action in the support of a players

11 In the static Bayesian game there is no history, so $\sigma_{i}: \Theta_{i}-\Delta\left(A_{i}\right) . V_{i}$ is the usual definition of expected utility, $V_{i}\left(\sigma, \tau, \theta_{i}\right)=\sum_{\theta} \tau\left(\theta \mid \theta_{i}\right) \sum_{a} \sigma(a \mid \theta) u_{i}(a, \theta)$. 
strategy must be an -best response. rather than just the strategy itself being an c-best response. This strengthening rules out the possibility of placing small probabilities on actions that lead to relatively low payoffs. This stronger conclusion turns out to be important in obtaining the absorption in Example 2 (see the discussion following Theorem 1). for instance.

Definition: Given $\tau$. a profile of strategies of the uncertain recurring game $\sigma$ plays $t$-like a profile of strategies of the realized static Bayesian game $\tilde{\sigma}$ at stage $t+1$ after history $h^{t}$. if there exists $\Theta^{\prime} \subset \Theta$ with $\tau\left(\Theta^{t}\right)>1-\epsilon$ such that $\sigma_{i}\left(\theta_{i}, h^{t}\right)$ is $c-c l o s e$ to $\tilde{\sigma}_{i}\left(\theta_{i}\right)$ for all $\theta_{i} \in \Theta_{i}^{*}$.

The above definition states that a set of strategies of the uncertain recurring game plays e-like a set of strategies of the true static Bayesian game if a large enough set of types

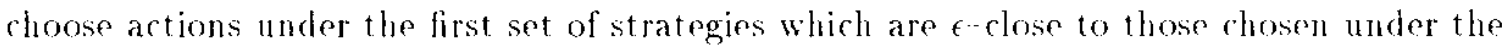
second set of strategies. This definition clearly implies that the overall distribution orer artions will be 6 -rlose under the two strategies. (For any $\delta>0$. there exists an $\epsilon>0$ such that if $\sigma$ plays, like $\tilde{\sigma}$. then $P_{\sigma}\left(a^{t+1} \mid \tau, h^{t}\right)$ is $\delta$-close to $P_{\sigma}(a \mid \tau)$.) It may still be that some types that occur with very low probability are playing different actions under the two strategies. This is illustrated in example 1.

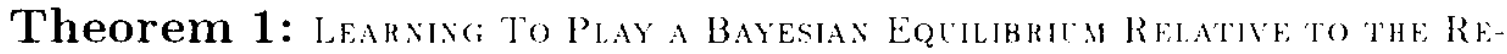
Aluzed Distribltion. ('onsider an uncertain recurring game with payoff sufficiont signals and an uncertain Baysian equilibrium $\sigma$ such that social learning implies private learning. For esery $\epsilon>0$ and almost every $\tau$. $h$ there is a time $T$ ' such that $\sigma$. at stage $t+1$ after

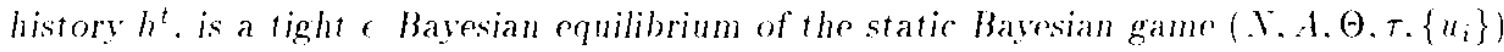
for rach $1 \geq T$. Moreover. for each $t \geq T$ there exists a Bayesian ergulibrinm $\tilde{\sigma}$ of the static

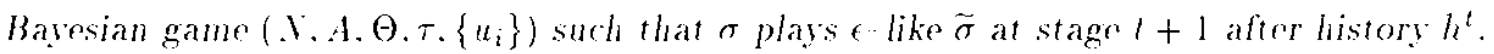

Proof: Fix any $\tau$ such that $\Gamma(\tau)>0$. It is sufficient to show that for any $(>0$ and almost every $h\left(P_{\sigma}^{\prime}\right.$ conditional on $\left.\tau\right)$ there is a time $T$ such that for each $t \geq T . \sigma\left(\cdot h^{t}\right)$ is a tight 6-Bayesian equilibrinm relative to $\tau$ and that there exists an Bayesian equilibrimm $\tilde{\sigma}$ of the static Bayesian game such that $\sigma$ plays $f-$ like $\widetilde{\sigma}$.

First, note that by Lemma 2, and social learning implies private learning. it follows that for almost every $h\left(P_{\sigma}\right.$. conditional on $\left.\tau\right)$ and any $\delta>0$ there exists $T$ such that if 
$t \geq T$. then

$$
\max _{\mathrm{s}^{t+1}}\left|P_{\sigma}\left(s^{t+1} \mid h^{t} \cdot \theta_{i}\right)-P_{\sigma}\left(s^{t+1} \mid \tau, h^{t} \cdot \theta_{i}\right)\right|<\delta
$$

for all $i$ and $\theta$ such that $\tau(\theta)>0$.

We now show the first conclusion of the theorem: for any $\epsilon>0$ and almost every $h$. there exists $T$ such that $\sigma\left(\cdot, h^{t}\right)$ is a tight $\epsilon$-Bayesian equilibrium relative to $\tau$ for each $t \geq T$. Civen the payoff sufficient signals. we can write the expected payoff to a player of type $\theta_{i}$ after history $h^{t}$ of some action $a_{i}$. given that all other players follow $\sigma$. as $V_{i}\left(a_{i}, \sigma, h^{t}, \theta_{i}\right)=\sum_{s^{t+1}} P_{\sigma}\left(s^{t+1} \mid h^{t}, \theta_{i}\right) \widehat{u}_{i}\left(a_{i}, \theta_{i}, s^{t+1}\right)$. where $\widehat{u}_{i}\left(a_{i}, \theta_{i}, s\right)=u_{i}\left(a_{i}, \bar{a}_{-i}, \bar{\theta}\right)$. for some $\bar{a} \cdot \bar{\theta}$ with $\bar{\theta}_{i}=\theta_{i}$. and $s=s_{\bar{i}, \bar{\theta}}$ (and $\hat{u}_{i}\left(a_{i}, \theta_{i}, s\right)$ is set arbitrarily if there is no such $\bar{a}, \bar{\theta}) .{ }^{12}$ Similarly, define $V_{i}\left(a_{i}, \sigma, \tau, h^{t}, \theta_{i}\right)=\sum_{s^{t+1}} P_{\sigma}\left(s^{t+1} \mid \tau, h^{t}, \theta_{i}\right) \widehat{u}_{i}\left(a_{i}, \theta_{i}, s^{t+1}\right)$. Then.

$V_{i}\left(a_{i}, \sigma, \tau, h^{t}, \theta_{i}\right)-V_{i}\left(a_{i}, \sigma, h^{t}, \theta_{i}\right)=$

$$
\sum_{s^{t+1}}\left[I_{\sigma}\left(s^{t+1} \mid \tau, h^{t} \cdot \theta_{i}\right)-P_{\sigma}\left(s^{t+1} \mid h^{t} \cdot \theta_{i}\right)\right] \widehat{u}_{i}\left(a_{i}, \theta_{i}, s^{t+1}\right)
$$

So. given the bounds on utility,

$$
\left|V_{i}\left(\sigma_{i}, \sigma, \tau, h^{t}, \theta_{i}\right)-V_{i}\left(a_{i}, \sigma \cdot h^{t}, \theta_{i}\right)\right| \leq M\left|\sum_{n}\left[I_{\sigma}\left(s^{t+1} \mid \tau \cdot h^{t} \cdot \theta_{i}\right)-P_{\sigma}\left(s^{t+1} \mid h^{t}, \theta_{i}\right)\right]\right| .
$$

Choose the of preceeding $(6)$ to be $\frac{(\# A) t}{2.1 f}$. and find $T$ accordingly. Thus. we know that $\left|V_{i}\left(a_{i}, \sigma \cdot \tau, h^{t}, \theta_{i}\right)-V_{i}\left(a_{i}, \sigma, h^{t}, \theta_{i}\right)\right| \leq t / 2$ for any $t \geq T$ and any $a_{i}$. Since $\sigma$ is an uncertain Bayesian equilibrium, and thus each $a_{i} \in \sigma_{i}\left(\theta_{i}, h^{t}\right)$ maximizes $l_{i}\left(a_{i}, \sigma . h^{t} . \theta_{i}\right)$, the above inequalities imply that each $a_{i}$ in the support of $\sigma_{i}\left(\theta_{i}, h^{t}\right)$ is an e best response relative to $\tau$.

Sext. We show the second conclusion of the theorem: for any $\mathrm{c}>0$ and almost every $h\left(l_{\sigma}\right.$-conditional on $\left.\tau\right)$ there exists $T$ such that for each $t \geq T$ there exists an Bayesian equilibrium $\tilde{\sigma}$ of the static Bayesian game such that $\sigma$ plays $\epsilon$ - like $\tilde{\sigma}$. Suppose to the contrary that there exists a positive measure of $h$ such that this does not hold. Pick any $h$ such that (6) holds and $\sigma$ does not play $\epsilon$-like any Bayesian equilibria $\widetilde{\sigma}$ of the static game. for infinitely many t's. Index the $\theta_{i}$ 's according to the positive integers and then for the first $\theta_{i}$ find a subsequence of the above $t$ 's such that $\sigma_{i}\left(\theta_{i}, h^{t}\right)$ converges to some $\bar{\sigma}_{i}\left(\theta_{i}\right)$. Then procend to do the same for each $\theta_{i}$ in the ordering. taking a further subsecuence each time.

12 Notien that in this defintion, the signal is predicted as if there were no deviation by $i$. Given the payoff sufficient signal condition, $i$ can couple this information with $a_{i}$ and $\theta_{i}$ to calculate the expected utility. 
Note that for some sufficiently large $t$ along a particular subseguence. $\sigma$ plays 6 -like $\bar{\sigma}$. (Take any finite set. $\Theta^{\prime} \subset \Theta$ with $\tau\left(\Theta^{*}\right)>1-\epsilon$ and then choose the subsequence corresponding to the last indexed $\theta_{i} \in \Theta_{i}^{r}$.) Thus, $\bar{\sigma}$ rannot be a Bayesian equilibrium relative to $\tau$. So. there exists $i, \theta_{i}$ and $\bar{a}_{i} \in A_{i}$ such that $V_{i}\left(\bar{a}_{i}, \bar{\sigma}, \tau, \theta_{i}\right)-V_{i}\left(a_{i}, \bar{\sigma} \cdot \tau, \theta_{i}\right) \geq a$ for some $a>0$ and any $a_{i}$ in the support of $\bar{\sigma}_{i}\left(\theta_{i}\right)$. where $V_{i}\left(a_{i}, \bar{\sigma}, \tau, \theta_{i}\right)=\sum_{s} I \frac{\sigma_{\sigma}}{\sigma}\left(s \mid \tau . \theta_{i}\right) \widehat{u}_{i}\left(a_{i}, \theta_{i}, s\right)$. For any $y>0$ there is some sufficiently large $T$ along a subsequence of the subsequence defined for $\theta_{i}$ such that $P_{\bar{\sigma}}\left(s \mid \tau, \theta_{i}\right)$ is $\nu$ - close to $P_{\sigma}\left(s \mid \tau, h^{t}, \theta_{i}\right)$ for $t \geq T$ along this subsequence. [This further subsequence is found by taking a funite subset of $\Theta_{-i}$ with large enough conditional probability (according to $\tau$ ) given $\theta_{i}$. Intersect the subsequences corresponding to these types $\theta_{-i}$ and $\theta_{i}$ so that $\sigma_{-i}$ converges to $\bar{\sigma}_{-i}$ for this arbitrarily large ( $\tau$ conditional on $\theta_{i}$ ) group of types $\theta_{-i}$. ) Thus. it follows that for sufficiently large $t$ along this subseruence, $V_{i}\left(\bar{a}_{i}, \sigma, \tau, h^{t}, \theta_{i}\right)-V_{i}\left(a_{i}, \sigma, \tau, h^{t}, \theta_{i}\right) \geq a / 2$ for some $a_{i}$ in the support of $\sigma_{i}\left(\theta_{i}, h^{t}\right)$. (For large enough $t$ on this subsequence. the support of $\bar{\sigma}_{i}\left(\theta_{i}\right)$ is a subset of the support of $\sigma_{i}\left(\theta_{i}, h^{t}\right)$.) By our earlier argument (that for any $\epsilon>0$ there exists large enough $T$ such that $\left|V_{i}\left(a_{i}, \sigma, \tau, h^{t}, \theta_{i}\right)-V_{i}\left(a_{i}, \sigma, h^{t}, \theta_{i}\right)\right|<c$ for any $1 \geq T$. $\theta_{i}$ and $\left.a_{i}\right)$. it follows that $V_{i}\left(\bar{a}_{i}, \sigma, h^{t}, \theta_{i}\right)>V_{i}\left(a_{i}, \sigma \cdot h^{t}, \theta_{i}\right)$ for some large enough $t$ and $a_{i}$ in the support of $\sigma_{i}\left(\theta_{i}, h^{t}\right)$. This is a contradietion, since $\sigma$ is an uncertain Bayesian equilibrium.

There are two conclusions to Theorem 1. First. after a sufficient time the actions that rach type of any player chooses in an uncertain Bayesian equilibrium are in fact $\epsilon$-best responses to the actions and types expected under the (unknown) realized type generating distribution. Notice that this first conclusion conld allow for actions which are different from those under a Bayesian equilibrimm of the static game. The second conclusion says that after a sufficient time play must. in fact. be close to that of a Bayesian equilibrium relative to the realized type generating distribution.

Notice that the static Bayesian equilibrium $\tilde{\sigma}$ to which play is close may vary from one stage to the next. This is due to the fact that players may condition on history and thus may be playing correlated actions across time. A very simple example is one where players play one equilibrium in even periods and another in odd periods.

With Theorem 1 in hand. let us revisit Example 2 (the Batte of the Sexes game with some naive best responders). When the $\tau$ described in Example 2 is the true distribution. we have the conclusion that rational players will play close to one of the profiles of Bayesian equilibrium strategies which are outlined in Example 2. ${ }^{13}$ Also. they must cloose actions

\footnotetext{
13 To apply Theorem 1. Iet naive best responders have utility 0 for any action combination.
} 
which are -best responses to the true dist ribution. In stages after (A.A) or (B.B) have been played, for $\epsilon$ small enough, since best responses are strict. this reguires that rational players exactly follow the equilibrium strategies of (A.A) or (B.B). respectively. Thus the absorbing nature of the Bayesian equilibrium is carried over to the uncertain Bayesian equilibrium.

Theorem 1 states that after some time an uncertain Bayesian equilibrium is a tight eBayesian equilibrium relative to the realized distribution. and that at each stage after that time it plays e like some Bayesian equilibrium relative to the realized distribution. Thus. every type is almost best responding relative to the true distribution and the aggregate actions play close to a true Bayesian equilibrium. Does this also mean that each type is alnost playing the same as in some Bayesian equilibrium? The answer is not necessarily if there is an infinite number of types. This is illustrated in the following example where there are always types whose strategies are not close to any of their Bayesian equilibrium strategies.

FXAMPLE 4.

There are two players, $N=\{1,2\}$. who each have two pure actions $\left(A_{i}=\left\{a_{i}, \bar{a}_{i}\right\}\right)$ available. Player 2 has two possible types. $\Theta_{2}=\left\{\theta_{2}, \bar{\theta}_{2}\right\}$. while player 1 has a countalle set of types represented by $\Theta_{1}=\{1.22 .3 \ldots\}$. Ltility is private valued and there is perfect monitoring ( signals reveal the actions chosen).

In the following tables. the first entry represents $u_{1}$ and the second $u_{2}$.

\begin{tabular}{cccccc} 
& \multicolumn{1}{c}{$\theta_{2}$} & & & $\bar{\theta}_{2}$ & \\
& $a_{2}$ & $\bar{a}_{2}$ & & $a_{2}$ & $\bar{a}_{2}$ \\
$a_{1}$ & $1+\left(\frac{1}{2}\right)^{\theta_{1}} .1$ & 0.0 & $a_{1}$ & $1+\left(\frac{1}{2}\right)^{\theta_{1}} .0$ & 0.1 \\
$\bar{a}_{1}$ & 0.1 & 1.0 & $\bar{a}_{1}$ & 0.0 & 1.1
\end{tabular}

Distributions over types are independent. There is only one distribution of player 1 types. which is deseribed by $\tau_{1}\left(\theta_{1}\right)=\left(\frac{1}{2}\right)^{\theta_{1}}$. There are two distributions over player 2 s types. $\tau_{2}$ and $\bar{\tau}_{2}$. These are such that $\tau_{2}\left(\theta_{2}\right)=1 / 2=\tau_{2}\left(\bar{\theta}_{2}\right)$. while $\bar{\tau}_{2}\left(\theta_{2}\right)=1 / 4$ and $\bar{\tau}_{2}\left(\bar{\theta}_{2}\right)=3 / 4$.

so that their behavior satisfies the premise of the theorem. We discuss this technique in Sections. 
Players are uncertain and $\Gamma\left(\tau_{1}, \tau_{2}\right)=1 / 2=I^{\top}\left(\tau_{1}, \bar{\tau}_{2}\right)$.

The Bayesian equilibria relative to the realized distributions are unique. Player 2 always follows the dominant strategy of $a_{2}$ if $\theta_{2}$ and $\bar{a}_{2}$ if $\bar{\theta}_{2}$. If the true distribution is $\tau$. then all types of player 1 should play $a_{1}$. If the true distribution is $\bar{\tau}$. then all types of player 1 should play $\bar{a}_{1}$.

In any uneretain Bayesian equilibrium of this uncertain recurring game, it is clear that player 2 will play the dominant strategy of $a_{2}$ if $\theta_{2}$ and $\bar{a}_{2}$ if $\bar{\theta}_{2}$. Player 1 's optimal strategy depends on the preceived (updated) distribution over types of player 2. After any history the updated $\Gamma$ will still place positive weight on both distributions.

Suppose that the realized distribution is $\tau$. Then after any history. any player 1 will update. but will still place some positive weight on $\bar{\tau}$. This means that there will exist some type $\theta_{1}$ whose best response to the anticipated distribution of actions of player 2 is to play $\bar{a}_{1}$. This is also true of all types larger than $\theta_{1}$. As learning takes place, an arbitrarily large measure of of player 1 's types will be choosing the correct best response of $a_{1}$. but there will always remain some types choosing $\bar{a}_{1}$.

Example 1 depends on an infinite number of types. It is clear that if there are only a finite number of types possible under $\tau$. then if we take $t$ to be small enough all types would have to be playing strategies -close to the Bayesian equilibrium strategies.

For games where the payoffs are type independent, that is, uncertainty is only strategic. we obtain the stronger conclusion that play converges to that of either a corretated or a Nash equilibrimm. This is made precise in the following corollary.

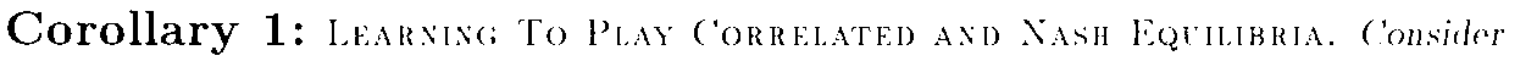
an uncertain recurring game with payoff sufficient signals and an uncertain Bayesian equilibrium $\sigma$ such that social learning implies private learning. Suppose also that $u_{i}$ is type independent for each $i$. For every $t>0$ and almost erery $\tau$.h there is a time $T$ such that for each $t \geq T$ there exists a correlated equilibrium $\tilde{\sigma}$ of the full information static game $\left(N,\left\{A_{i}\right\},\left\{u_{i}\right\}\right)$ such that $\sigma$ plays like $\tilde{\sigma}$ in stage $t+1$ after history $h^{t}$. Furthermore. if player types are indepedent under $\tau$. then $\tilde{\sigma}$ is a Nash equilibrium.

Proof: Consider the Bayesian equilibrium from Theorem 1. (iiven the type independence 
of $u_{i}$ there is a correlated equilibrium ( $\tau$ with information $\theta_{i}$ ) which plays exactly like that equilibrium. If types are independent. then this is a Nash equilibrium.

In the above corollary, sorjety is learning to "coordinate" on what to play in a game. For instance in the case where types are independent under $\tau$ and there are several Nash equilibria to a game. then they will learn to play so that all players will coordinate on a Nash equilibrimm at cach stage.

In some cases. the results of the corollary can be thought of as a purification. For instaner. if there is a mixed strategy equilibrium to the game, then players would play so that the perceived distribution matches the mixing. even though the players themselves may be choosing pure strategies.

We now examine failures of convergence due to violations of the conditions nsed in Theorem 1.

\section{Social Versus Private Equilibrium}

Example 5 shows the importance of payoff sufficient signals. Example 6 illustrates the role of the sorial learning implies private learning rondition.

\section{Examplt: 5. Payoll Sufficient Signals: Affirmative Action Revisited.}

There are two players. $Y=\{1.2\}$. Player 1 has two pure actions $A_{1}=\left\{a_{1} \cdot \bar{a}_{1}\right\}$ and player 2 has only one action $A_{2}=\left\{a_{2}\right\}$. Player 1 has one possible type. $\Theta_{1}=\left\{\theta_{1}\right\}$. While player 2 has two possible types. $\Theta_{2}=\left\{\theta_{2}, \bar{\theta}_{2},\right\}$. All types are rational. There is perfect monitoring (signals reval actions).

One distribution $\tau$ is such that $\tau\left(\theta_{1}, \theta_{2}\right)=.8$. while another distribution $\bar{\tau}$ is such that $\bar{\tau}\left(\theta_{1} \cdot \theta_{2}\right)=2$. Players are uncertain and $\Gamma(\tau)=1 / 2=I^{\prime}(\bar{\tau})$. Thus. player l's initial uncertainty treats $\theta_{2}$ and $\bar{\theta}_{2}$ as bring equally likely.

In the following tables. the first entry represents $u_{1}$ and the second $u_{2}$.

$\begin{array}{cccc} & \theta_{2} & & \bar{\theta}_{2} \\ & a_{2} & & a_{2} \\ a_{1} & 1.0 & & -2.0 \\ \bar{a}_{1} & 0.0 & a_{1} & 0.0\end{array}$


Consider constant strategies $\sigma$ where player 1 always plays $\bar{a}_{1}$ and player 2 always plays $a_{2}$. The strategies $\sigma$ form an uncertain Bayesian equilibrium of the above uncertain recurring game. Both $\tau$ and $\bar{\tau}$ lead to the same distribution over actions under $\sigma$ and so $\Gamma$ is never updated, even if the payoffs are revealed (but not if the type of player 2 becomes known). However. if player 1 knew that the true distribution was $\tau$. then his or her unique best response would be $\bar{a}_{1}$.

In the above example there is perfect monitoring. payoffs are made known. and social learning implies private learning. The prescribed strategies form an equilibrium of the unertain recurring game, and yet these strategies are not close to any Bayesian eguilibrium of the game where the true distribution is known. The difficuly lies in the fact that player 2 is ype is important in determining player 1 's payofs, and can never be learned from a history of signals where player 1 only plays $\bar{a}_{1}$.

The following example. shows the importance of the social implies private learning condition. In this example players have private values, there is perfect monitoring. and soriety learns to perfectly predict future signals. However. players never learn which of two coordination games they are playing.

Example 6. Social sersus Priate Predictions.

There are two players. $X=\{1,2\}$. who each have two pure actions $\left(A_{i}=\left\{a_{i}, \bar{a}_{i}\right\}\right)$ available. Each player has two possible types, $\Theta_{i}=\left\{\theta_{i}, \bar{\theta}_{i}\right\}$. All types of all players are rational. There is perfect monitoring. One distribution $\tau$ is such that players' types are perfectly correlated: $\tau\left(\theta_{1}, \theta_{2}\right)=1 / 2=\tau\left(\bar{\theta}_{1}, \bar{\theta}_{2}\right)$. Another distribution. $\bar{\tau}$. has the opposite correlation structure: $\bar{\tau}\left(\theta_{1}, \bar{\theta}_{2}\right)=\bar{\tau}\left(\bar{\theta}_{1}, \theta_{2}\right)=1 / 2$. Players are uncertain and $\Gamma(\tau)=1 / 2=\Gamma(\bar{\tau})$. 
In the following tables. the first entry represents $u_{1}$ and the second $u_{2}$.

$\begin{array}{cccccc} & \theta_{1}, \theta_{2} & & & & \bar{\theta}_{1}, \bar{\theta}_{2} \\ & a_{2} & \bar{a}_{2} & & a_{2} & \bar{a}_{2} \\ a_{1} & 1.1 & 0.0 & a_{1} & 1.0 & 0.1 \\ \bar{a}_{1} & 0.1 & 1.0 & \bar{a}_{1} & 0.0 & 1.1 \\ & & & & & \bar{\theta}_{1}, \theta_{2} \\ & \theta_{1} \cdot \bar{\theta}_{2} & & & a_{2} & \bar{a}_{2} \\ & a_{2} & \bar{a}_{2} & & 1.1 & 0.0 \\ a_{1} & 1.0 & 0.1 & a_{1} & 0.1 & 1.0 \\ \bar{a}_{1} & 0.0 & 1.1 & \bar{a}_{1} & 0.1\end{array}$

Consifler constant strategies $\sigma$ which are an even mixing over $a_{1}$ and $\bar{a}_{1}$. for each type of player 1 and every history. Let each type of player 2 play his or her strictly dominant aetion $\left(\theta_{2}\right.$ plays $a_{2}$. while $\bar{\theta}_{2}$ plays $\left.\bar{a}_{2}\right)$. The st rategies $\sigma$ form an uncertain Bayesian equilibrium of the described recurring game. Both $\tau$ and $\bar{\tau}$ lead to the same distribution over actions under $\sigma$ and so $I$ is never updated. However. if player $1 \mathrm{knew}$ which of $\tau$ or $\bar{\tau}$ was realized. then conditional on his or her type player 1 would also know the type of player 2 and thus what action player 2 would take. Thus. an even mixing is not part of a Bayesian equilibrium strategy for player 1 when $\tau$ is known.

In the above examplo, there is perfect monitoring (signals conlel eren show the mixture (hosen). and players correctly predict public signals and strategies. Ilayers also have private values. However. social learning does not imply private learning and player l's actions conditional on his or her type are not part of a Bayesian equilibrim under the true distribution. The preseribed strategies form an equilibrium of the uncertain recurring game. and vet these strategies are not close to aly Bayesian equilibrium of the game where the true distribution is known.

Examples 5 and (; present us with an inferesting phenomenon. There are situations where the predietions of an outside observer converge and are completely accurate concerning the actions which will be chosen in a game. Yet the players in the game have not vet learned all that they might like to concerning the uncertainty in the game and are not necossarily playing an equilibrinu of the "true" game. 


\section{Learning to Play Perfectly}

In situations where the sufficient conditions for convergence to Bayesian equilibrium are met. it will artually be quite natural to expect convergence to a refinement of Bavesian equilibrium. For instance, if a player is never completely certajn of the actions that the other players might choose. then that player should never play a weakly dominated action. This means that convergence will actually be to an undominated Bayesian equilibrium of the static game. If. in addition. players types are drawn independently, then convergence will be to that of a tembling hand perfect equilibrium.

We will first present a result concerning convergence to trembling hand perfect equilibrium, and then discuss convergence to an undominated Bayesian equilibrium that may not be trembling hand perfect. To make these statements precise consider the following definitions.

Definition: A distribution $\Gamma$ satisfies full subjective uncertainty relative to a profile of strategies $\sigma$ if $P_{\sigma}\left(a_{-i}^{t+1} \mid h^{t} . \theta_{i}^{t}\right)>0$ for all $i, t, a_{-i}^{t+1}$, and almost every $h^{t} . \theta_{i}^{t}$.

Definition: A distribution $I$ satisfes full independence if there exist $M_{1}\left(\Theta_{1}\right) \ldots M_{N}\left(\Theta_{N}\right)$ (sets of distributions over each $\Theta_{i}$ ) and corresponding distributions $\Gamma_{i}$ over each $M_{i}\left(\Theta_{i}\right)$ such that $\{\tau \mid \Gamma(\tau)>0\}=\mathrm{II}_{i}, H_{i}\left(\Theta_{i}\right)$ and $\Gamma(\tau)=I_{i} \Gamma_{i}\left(\tau_{i}\right)$ for any $\tau$ such that $\Gamma(\tau)>0$.

We define trembling hand perfect Bayesian equilibrium arcording to Selten $(1975) .{ }^{14}$

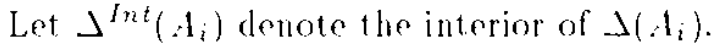

Definition: $\bar{\sigma}$ is a trembling hand perfect Bayesian equilibrium of the static Bayesian game

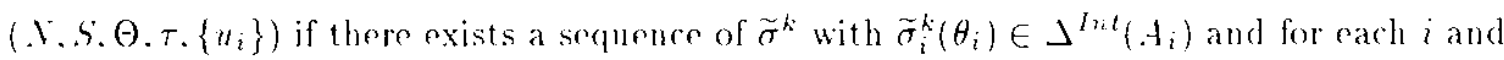
$\theta_{i}$ there exists a subsequence of the $k$ 's such that $\tilde{\sigma}_{i}^{k}\left(\theta_{i}\right)-\bar{\sigma}_{i}\left(\theta_{i}\right)$ and $\bar{\sigma}_{i}\left(\theta_{i}\right)$ is a best response to $\tilde{\sigma}_{-i}^{k}$ along the subsequence.

If there are only a finite number of types, then the mention of subsequeness in the definition is inconsequential as we can intersect them to find a single sequence. and the definition is equivalent to Selten's. Here we need to apply the definition to infinite numbers

1.4 A trembling hand perfect Bayesian equilibrium is thus distinct from the notion of perfect Bayesian equilibrium defined by Fudenberg and Tirole (1991) for extensive form games. 
of players (viewing each player as an agent, taking the agent normal form corresponding to the Bayesian game) which accounts for the need to make explicit use of subsequences. This defuntion allows us to capture situations such as Example t. where players behavior converges at different rates.

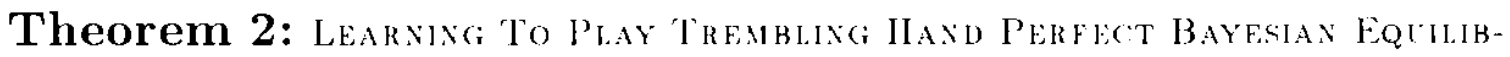
RItu. Consider an uncertain recurring game such that $u_{i}$ is prisate whed. there is perfect monitoring (signals reveal actions). and $\mathrm{T}$ is fully independent. If $\sigma$ is an uncertain Bayesian equilibrium such that I has full subjective uncertainty relative to $\sigma$, then for erery $\epsilon>0$ and almost every $\tau$, h there is a time $T$ such that for each $t \geq T$ there exists a trembling hand perfect Bayesian equilibrium $\widetilde{\sigma}$ of the static Bayesian game $\left(\mathcal{X}, S, \Theta, \tau,\left\{C_{i}\right\}\right)$ such that $\sigma$ playse like $\tilde{\sigma}$ at stage $t+1$ after history $h^{t}$.

PROOF: Fix any $\epsilon>0$ and $\tau$ such that $\Gamma(\tau)>0$. It is sufficient to show that for almost every $h(P$-conditional on $\tau)$ there is a time $T$ such that for each $t \geq T$ there exists a trembling hand perfect Bayesian equilibrium $\widetilde{\sigma}$ of the static Bayesian game $\left(\mathcal{N}, \mathcal{A}, \Theta, \tau,\left\{u_{i}\right\}\right)$ such that $\sigma$ plays e-like $\tilde{\sigma}$

Suppose that there exists a positive measure of $h$ such that $\sigma$ does not play $t$ - like any trembling hand perfect equilibrium of the stage game for an infinite subsequenee of l's. By Lemma 2 and perfoct monitoring. we can find such an $h$ such that for any $d$. $\max _{\mathrm{a}}\left|P_{\sigma}\left(a \mid h^{t}\right)-P_{\sigma}\left(a \mid \tau, h^{t}\right)\right|<\delta$ for all $t$ sufficiently large.

Index the $\theta_{i}$ 's (according to the positive integers) and then for the first $\theta_{i}$ find a subsequence of the above t's such that $\sigma_{i}\left(\theta_{i}, h^{t}\right)$ converges to some $\bar{\sigma}_{i}\left(\theta_{i}\right)$. Then procend to do the same for rach $\theta_{i}$ in the ordering. laking a further subsequence each time. For any $l>0$ and sufficiently large $t$ along a particular subsequence (similar to the one defuned in the proof of Theorem 1). $\sigma$ plays $y$-like $\bar{\sigma}$. Thus. $\bar{\sigma}$ cannot be a trembling hand perfect Bayesian equilibrium relative to $\tau$.

We now show that for earh $i$ and $\theta$ such that $\tau(\theta)>0$. there exists a subsequence of the $t$ s such that each $a_{i}$ in the support of $\bar{\sigma}_{i}\left(\theta_{i}\right)$ is a best response to $I_{\sigma}^{\prime}\left(a_{-i} \mid h^{t}\right)$ along that subsequence. For any $i$ and $\theta$ such that $\tau(\theta)>0, \sigma_{i}\left(\theta_{i} . h^{t}\right)$ converges to $\bar{\sigma}_{i}\left(\theta_{i}\right)$ along the subsequence of $t$ 's used in the definition of $\bar{\sigma}$. Thus, given the finite action space. the support of $\sigma_{i}\left(\theta_{i} . h^{t}\right)$ contains the support of $\vec{\sigma}_{i}\left(\theta_{i}\right)$ far enough along the subsequence. By full independence, private values, and the definition of uncertain Bayesian equilibrium we know that $\sigma_{i}\left(\theta_{i}, h^{t}\right)$ is a best response to $P_{\sigma}\left(a_{-i} \mid h^{t}\right)$. Thus. far enough along 1 he subsequence. every $a_{i}$ in the support of $\bar{\sigma}_{i}\left(\theta_{i}\right)$ is a best response to $P_{\sigma}\left(a_{-i} \mid h^{t}\right)$. which establishes our desired conclusion. 
To complete the proof of the theorem, we construct a sequence $\tilde{\sigma}^{t}$ such that $\tilde{\sigma}^{t}\left(\theta_{i}\right) \in$ $\Delta^{I n t}\left(A_{i}\right)$ and $\tilde{\sigma}^{t}\left(\theta_{i}\right)-\bar{\sigma}_{i}\left(\theta_{i}\right)$ for each $\theta_{i}$. and $P_{\sigma^{t}}(a \mid \tau)=P_{\sigma}\left(a \mid h^{t}\right)$. The construction of this sequence completes the proof of the theorem since it follows from above (and full independence) that every $a_{i}$ in the support of $\bar{\sigma}_{i}\left(\theta_{i}\right)$ is a best response to $\tilde{\sigma}^{t}$ far enough along a subsequence. This is a contradiction since our supposition implied that $\bar{\sigma}$ is not a trembling hand perfect Bayesian equilibrimm.

To construct the sequence $\tilde{\sigma}^{t}$, we first construct an auxillary secquence $\hat{\sigma}_{i}^{t}$ for each $i$ as follows. Order the set $A_{i}$. Consider the first $a_{i} \in A_{i}$ such that $P_{\bar{\sigma}}\left(a_{i} \mid \tau\right)>P_{\sigma}^{\prime}\left(a_{i} \mid h^{t}\right)$ and identify the set $\bar{\Theta}_{a_{1}}$ such that $\bar{\sigma}\left(a_{i} \mid \theta_{i}\right)>P_{\pi}\left(a \mid h^{t}\right)$ iff $\theta_{i} \in \bar{\Theta}_{a_{1}}$. Find $x_{n}^{t}$ such that

$$
\sum_{\theta_{1} \notin \bar{q}_{a_{1}}} \tau\left(\theta_{i}\right) \bar{\sigma}_{i}\left(a_{i} \mid \theta_{i}\right)+\sum_{\theta_{1} \in \overline{(}_{a_{1}}} \tau\left(\theta_{i}\right)\left(\max \left[\bar{\sigma}_{i}\left(a_{i} \mid \theta_{i}\right)-x_{a_{1}}^{t} \cdot 0\right]\right)=P_{\sigma}\left(a_{i} \mid h^{t}\right)
$$

For $\theta_{i} \notin \bar{\Theta}_{n_{i}}$ let $\hat{\sigma}^{t}\left(a_{i} \mid \theta_{i}\right)=\bar{\sigma}_{i}\left(a_{i} \mid \theta_{i}\right)$ and for $\theta_{i} \in \bar{\Theta}_{a_{i}}$ let $\widehat{\sigma}^{t}\left(a_{i} \mid \theta_{i}\right)=\max \left[\bar{\sigma}_{i}\left(a_{i} \mid \theta_{i}\right)-x_{n_{i}}^{t}, 0\right]$. (It is clear from its definition that $x_{t_{1}}^{t} \in[0.1]$.) Now proceed to the next $a_{i}^{\prime}$ in the ordering over $A_{i}$. except instead of using $\bar{\sigma}\left(a_{i}^{\prime} \mid \theta_{i}\right)$ as the base. we use $\bar{\sigma}\left(a_{i}^{\prime} \mid \theta_{i}\right)+\left[\bar{\sigma}_{i}\left(a_{i} \mid \theta_{i}\right)-\hat{\sigma}_{i}^{t}\left(a_{i} \mid \theta_{i}\right)\right]$ (thus ensuring that the base strategies still add to one). This process is iterated ${ }^{15}$

Define $\tilde{\sigma}^{t}\left(\theta_{i}\right)$ by

$$
\tilde{\sigma}_{i}^{t}\left(\theta_{i}\right)=\left(1-\lambda^{t}\right) \hat{\sigma}^{t}\left(\theta_{i}\right)+\lambda^{t} P_{\sigma}\left(a_{i} \mid h^{t}\right)
$$

where $\lambda^{t}>0$ is a sequence converging to 0 .

By full subjective uncertainty. we know that $\tilde{\sigma}^{t}\left(a_{i} \mid \theta_{i}\right) \in J^{\text {Int }}\left(A_{i}\right)$ for each $\theta_{i}$. We also know that by construction $P_{\sigma^{t}}(a \mid \tau)=P_{\sigma}^{\prime}\left(a \mid h^{t}\right)$ and so $P_{\sigma^{t}}(a \mid \tau)=I_{\sigma}^{\prime}\left(a \mid h^{t}\right)$. The last thing to show is that in a subsequence (of the previously defined subsequence) for each $\theta_{i}$. $\widetilde{\sigma}^{t}\left(\theta_{i}\right) \rightarrow \bar{\sigma}_{i}\left(\theta_{i}\right)$. Civen that $\lambda^{t}-0$. we need to show that $\hat{\sigma}^{t}\left(\theta_{i}\right) \rightarrow \bar{\sigma}_{i}\left(\theta_{i}\right)$. Without loss of generality assume that $P_{r}\left(a \mid h^{t}\right)$ converges to $\bar{P}(a)$ along the sequence of $t$ s. (Given the finiteness of $A$ we can choose an appropriate subsequence where this is true $)$. Since $I_{\sigma}\left(a \mid h^{t}\right)$ converges to $\bar{P}(a)$ along the sequence of $t$ 's, and since for any $l>0$. far enough along particular subsequences $\sigma$ plays $\nu$-like $\bar{\sigma}$. it follows that far enough along these particular subsequences we can make $P_{\sigma}\left(a \mid h^{t}\right)$ arbitrarily close to $P_{\sigma}^{\prime}(a \mid \tau)$. (See the comment after the definition of plays $\epsilon$ like). Thus $\bar{P}(a)=P_{\bar{\sigma}}(a \mid \tau)$ and so $P_{\sigma}\left(a \mid h^{t}\right)$ converges to $P_{\bar{\sigma}}(a \mid \tau)$. This implies that $x_{a,}^{t} \rightarrow 0$ for each $a_{i}$ in the construction of $\hat{\sigma}^{t}$. and so $\hat{\sigma}^{t}\left(\theta_{i}\right) \rightarrow \bar{\sigma}_{i}\left(\theta_{i}\right)$.

15 Once the distribution over an $a_{i}$ has been adjusted clownward so that $P_{\hat{\sigma}^{\prime}}\left(a_{i} \mid \tau\right)=$ $P_{\sigma}\left(a_{i} \mid h^{t}\right)$, it is not adjusted any further. If the last $a_{i}^{\prime}$ is the seguence is adjusted down. it must be that some previous $a_{i}^{\prime \prime}$ was such that $P_{\hat{\sigma}^{t}}\left(a_{i}^{\prime \prime} \mid \tau\right)<P_{\sigma}\left(a_{i}^{\prime \prime} \mid h^{t}\right)$. and the wright taken off action $a_{i}^{\prime}$ can be transferred to $a_{i}^{\prime \prime}$. in such a process. each action is adjusted downward at most once and when there are no actions to adjust downward the distributions must be equal. 
Again, in sitnations where preferences are type independent. we obtain a stronger conclusion.

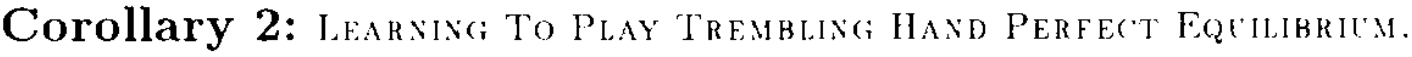

Consider an uncertain recurring game such that there is perfect monitoring and an equilibrium $\sigma$. Suppose also that $u_{i}$ is type independent. If $\Gamma$ is fully independent and has full subjective uncertainty relative to $\sigma$. then for every $\mathrm{c}>0$ and almost every $\tau$. h there is a time $T$ such that for each $t \geq T$ there exists a trembling hand perfect equilibrium $\tilde{s}$ of the full information static game $\left(X, A,\left\{u_{i}\right\}\right)$ such that $\sigma$ plays $6-l i k e \tilde{s}$ at stage $t+1$ after history $h^{t}$.

ProOf: This follows from Theorem 2 and corollary 1.

\section{The Role of Full Independence in Convergence to Perfect Equilibria}

If the full independence condition in Theorem 2 (and corollary 2) is not satisfied, but there is full subjective uncertainty, then play still converges to a refinement of Bayesian Nash equilibrium. Notice that if a player has uncertainty which allows for every action combination of the other players, then any best response to that player's beliefs about actions of other players has to be an undominated action. Thus, in situations where play converges to a Bayesian equilibrium play and there is full subjective uncertainty play will actually converge to an undominated Bayesian equilibrium. The following theorem is then an obvious consequence of Theorem 1.

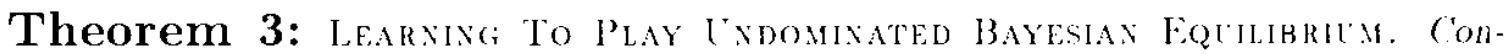
sider an uncertain recurring game with payoff sufficient signals and an equilibrium $\sigma$ such that social learning implies private learning. If $\Gamma$ has full subjective uncertainty relative to $\sigma$. then for every $t>0$ and almost every $\tau$.h there is a time $T$. such that for each $t \geq T$ there exists an undominated Bayesian equilibrium ${ }^{16} \tilde{\sigma}$ of the static Bayesian game (N.S. Q.T. $\left.\left\{C_{i}\right\}\right)$ such that $\sigma$ plays , like $\tilde{\sigma}$ at stage $t+1$ after history $h^{t}$.

In the case of $X=2$, the set of undominated Nash rquilibria is the same as the set of trembling hand perfect equilibria (see van Damme (1987)). It is not clear to what extent

${ }^{16}$ Undominated is defined in the weak sense. where $a_{i}$ dominates $a_{i}^{\prime}$ for $\theta_{i}$ if it yields at least as high a payoff (and sometimes higher) no matter the actions and the types of the other players (considering only those types which receives positive probability conditional on a player's type). 
this result generalizes to Bayesian eguilibrium. When $X \geq 3$, however, this equivalence is broken and play does not necessarily converge to trembling hand perfect equilibrium play. This is illustrated in Example 7 , below. Different players end up with different beliefs after observing the same history because conditioning on their types may lead them to different updating. Thus there is no common set of trembles justifying their actions. ${ }^{17}$

EXAMPLE $て$.

There are three players whose payoffs are type independent. Actions and payoffs represented below. Player 1 chooses a row, player 2 chooses a column, and player 3 chooses a matrix.

\begin{tabular}{ccccccc} 
& & $l$ & & \multicolumn{3}{c}{$r$} \\
& $L$ & $M$ & $R$ & $L$ &.$H$ & $R$ \\
$t$ & 1.2 .1 & 2.0 .1 & 0.0 .0 & 1.2 .1 & 2.0 .0 & 0.0 .2 \\
$b$ & 1.1 .1 & 0.0 .1 & 1.0 .0 & 1.2 .1 & 0.0 .0 & 1.0 .2
\end{tabular}

Here all full information trembling hand perfect equilibria result in payoffs of $1.2 .1 .^{18}$ The set of undominated Nash equilibria is larger. consisting of those where player 1 chooses any mixture of $t$ and $b$. player 2 plays $L$. player 3 chooses any mixture of $l$ and $r$.

Consider. the undominated Nash equilibrium (b.L.l). which is not trembling hand perfect. Let us describe initial uncertainty which leads play to converge to b. L.l.

$L$ is strictly dominant for 2 so any equilibrium will have 2 play $L$. To justify $b$ for player 1. she must believe that 2 will play $R$ with at least twice as high a probability as 2 will play $M$. To justify $/$ for player 3. he must believe that 2 will play $R$ with at most half as high a probability as 2 will play $.1 .^{19}$ Such beliefs can arise in the learning enviroment. as players upelate based on their own types. before choosing an action. This may lead them to different upelating for players 1 and :3.

17 Similar considerations for different types of the same player are what mat break the equivalence between undominated Bayesian equilibrium and trembling hand perfect Bayesian equilibrium for $.1=2$.

is The perfect equilibria are any mixture between $t$ and $b$ coupled with $L$.r: and $t . I$, coupled with any mixture of $l$ and $r$.

19 This is not a perfect equilibrium since there is no single set of trembles for player 2 which would justify both $l$ 's and 3 's actions simultaneously. 
For instance. let $\Theta_{1}=\left\{\theta_{1}^{r}, \theta_{1}^{m}\right\}, \Theta_{2}=\left\{\theta_{2}^{r}, \theta_{2}^{H}, \theta_{2}^{R}\right\}$, and $\Theta_{3}=\left\{\theta_{3}^{r}, \theta_{3}^{m}\right\}$. $\theta_{i}^{r}$ is interpreted to be a rational type who has payoffs given in the above table. The other types are irrational and have flat preferences, so that their payoff from any action combinations is 0 . Define strategies as follows. $\sigma_{i}\left(\theta_{i}^{r}\right)$ is the prescribed part of $(b . L . l) . \sigma_{i}\left(\theta_{i}^{m i}\right)$ is a uniform mixing across $i$ 's actions. $\sigma_{2}\left(\theta_{2}^{M}\right)$ plays $M$ with probability $3 / 4$ and $R$ with probability $1 / 4$. $\sigma_{2}\left(\theta_{2}^{R}\right)$ plays $M$ with probability $1 / 4$ and $R$ with probability $3 / 4$.

Consider the following distributions: $\tau\left(\theta^{r}\right)=1, \bar{\tau}\left(\theta_{1}^{m}, \theta_{2}^{M} \cdot \theta_{3}^{r}\right)=1, \widetilde{\tau}\left(\theta_{1}^{r}, \theta_{2}^{R}, \theta_{3}^{m}\right)=1$. and $\widehat{\tau}\left(\theta_{1}^{m}, \theta_{2}^{r}, \theta_{3}^{m}\right)=1$. Initial uncertainty, $\Gamma$, has weight $\frac{3}{4}$ on $\tau$ and $\frac{1}{12}$ on each of the remaining distributions.

When $\tau$ is the true (realized) distribution only (b. L.l) will ever be played. C'onditioning on histories, players will put weight increasingly on $\tau$ and decreasingly on the others, since no other distribution can lead to b. L.l. The weight on these other distributions will become arbitrarily small, but never disappear. Once player 1 sees that she is $\theta_{1}^{r}$. she will update ruling ont $\bar{\tau}$ and $\hat{\tau}$. but retaining $\tilde{\tau}$. This justifies plaving $b$. Similarly, once player 3 sees that he is $\theta_{3}^{r}$. he will update ruling out $\tilde{\tau}$ and $\hat{\tau}$. but retaining $\bar{\tau}$. This justifies playing $l$.

\section{Bounded Rationality, Long Lived Players, and Stochastic Games.}

Several easy technical modifications cmable one to extend the previous results on uncertain recurring games with rational players to significantly richer models. Here, we briefly describe such morlifications.

First. we describe the incorporation of "irrational" players. A player type following some irrational strategy can be made game theoretically rational (Bayesian. expected utility maximizing) by modifying their utility function. as done for example in hreps. Milgrom. Roberts, and Wilson (1982) and Aumann (1992). We use an easier method (cheaper trick?) by endowing such irrational players with flat utility functions. i.e.. utility functions that are independent of types and actions. " ('learly, any behavior is a best response given such preferences. This approach was used following Theorem 1 in discussing the Battle of the sexes game Example 2, which had rational types. as well as naive best responding types who mimicked the action of the previous period opposite player. This example fully fits the uncertain Bayesian recurring game setup once we modify the payoff to be uniformly 0 for

20 For some "irrational" type $\hat{\theta}_{i}$ let $u_{i}(a, \theta)=0$ for all $a$ and any $\theta$ such that $\theta_{i}=\hat{\theta}_{i}$. 
any player whose realized type is nbr. We then consider an uncertain Bayesian equilibrium where nbr types follow their mimicking strategy. Our results can then be applied and the asymptotic results stated in Example 2 follow. In Jackson and halai (1995a) we used this technique to explore Kreps-Milgrom-Roberts-Wilson results concerning chain stores in a recurring setting.

Second. the uncertain recurring model is easily modified to accomodate a recurring version of a stochastic game. Following Shapley (1953). a stochastic $n$ person game consists of the usual individual sets of actions $\left(A_{i}\right)$. but with individual utility functions $u_{i, q}$ which depend on a publicly known state $q$ selected from a finite set of states $Q$. A fixed. known collection of stochastic transition rules $\left(\eta_{n}\right)$. with $\eta_{n} \in \Delta(Q)$, describe the movement anong states according to the vector of actions chosen in the last stage. Thus. the game starts in an initial known state $q^{1}$ and players choose actions $a^{1}$ and receive payoffs $u_{i, q^{1}}\left(a^{1}\right)$. Based on these actions. nature randomly chooses a new state $q^{2}$ according to $\eta_{2^{1}}\left(q^{2}\right)$. which is publicly announced before the second stage is played. This process is repeated.

A stochastic recurring game has only one modification. For each stage a new set of players is selected. To represent such a stochastic recurring game as a special case of the uncertain recurring games presented in this paper. model nature as an extra player, labeled 0 . who has a flat ulility function. Formally, natures actions are the set of states $Q$. and the utility of each player $i \neq 0$, when nature chooses $q$ and the remaning players choose a. $u_{i}(a . q)$. is simply defined to be the corresponding stochastic game utility $u_{i, q}(a)$. To endow the $t+1$ stage players with the knowlerge of which state nature will choose in stage $t+1$. model the public signal coming out of stage $t$ to be $s^{t}=\left(a^{t} . q\right)$ where $q$ is randomy chosen according to $\eta_{n^{2}}$, and then nature follows a strategy that plays q (from $s^{t}$ ) at time $t+1$. Notice that this construction can be modified so that the signals only partly reval q. and nature randomizes after observing the signal allows for substantially richer types of stochastic games with incomplete and imperfect information.

Finally, our recurring game results can be useful in modeling situations where some players are long lived. For instance, player 1 may be infinitely lived with a type drawn during the first period and fixed forever. while the remaining players are new each period and have their types drawn in each period. Such a game can be modeled by replacing the infinitely lived player I with a series of plavers (1.t) who may be thought of as player 1 's 
agents and who have flat utility functions. Further. let the type generating distributions $\tau$ be such that a single type of player one is chosen with probability one, and incorporate into $\Gamma$ the original prior probability that the long lived player 1 is of the type described by $\tau .^{21}$ The type of player 1 is then drawn at time 0 (when $\tau$ is drawn) and remains fixed thereafter. 'To such a game we can apply our results to conclude that players 2 through $n$ will learn to best respond, each in his or her own period t. to the strategy of player $(1, t)$. Then. we can conclude that whatever the type of the long lived player 1. late recurring short lived players will be approximately best responding to plaver l's actual strategy.2.2

\section{Concluding Remarks}

We close with comments on two assumptions that we have maintained in our analysis. and a comment on existence.

First. we have assumed that the initial uncertainty. $\Gamma$. is the same for all players. The model is easily adapted to allow the uncertainty. $T$, to depend on a player's type. The learning results (Lemmas 1 and 2 ) will still hold for each type separately provided that each types beliefs are absolutely continuous with respect to the true uncertainty. The change in the results due to type dependent uncertainty is that there may be no uniform rate of convergence when the set of types is infinite. This affects the conclusions of Theorems 1 and 3 only in that some arbitrarily small measure of types may not have converged to be e-best responding to the true distribution at any time. The results of playing 6 -like a true Bayesian equilibrium (or undominated Bayesian equilibrium) would remain unchanged since it only required convergence for a finite number of types to begin with. The convergence to trembling hand perfect Bayesian equilibrium is affected more substantially (except for 2 player games). For instance. two players unght have different beliefs about the distribution of actions that a third player might choose. This could allow convergence to an undominated Bayesian equilibrium that is not trembling hand perfect. along similar lines to Example $i$.

Second. we have limited attention to a countable set of types and a countable set of distributions over those types. The method of proof that we have used relies on these

21 For each $\tau$ there exists $\theta_{1}$ such that if $\tau(\bar{\theta})>0$. then $\bar{\theta}_{1}=\theta_{1}$.

22 Player l's best response to the short lived players. may involve long term goals which are not captured in the period by period maximization of the recurring setup. Thus. a hybrid analysis of rational learning by players with lives of varying lengths is needed to fully address situations which are not strictly repeated. nor strictly recurring. 
assumptions. Noreover, results by Jordan (1993) in the context of boundedly rational learning suggests that in general there may be difficulties in learning to play mixed strategy equilibria when these assumptions are relaxed. At this time, we do not have much of an idea of how the results extend when these assumptions are violated, but view it as an important issue for future research. ${ }^{23}$

Finally, let us say something about the existence of an uncortain Bayesian equilibrium. ${ }^{24}$ If the set of types receiving positive probability given the initial uncertainty $I$ is finite (even if the support of $\Gamma$ over pessible $\tau$ 's is not), then existence can be established using standard results. The first stage is simply a finite Bayesian game (with a possibly inconsistent prior) for which an equilibrium exists. Csing the first stage equilibrium st rategies for ach type, the second period upelating is then clearly defined and the existence of equilibrium strategies for the second period. conditional on any of the fuite possible histories. can be established. and so on. If there is an infinite set of possible types under the initial uncertainty, then the type-agent representation of the first stage game will have an infinite number of players. as possibly will subsequent stages. In such a case the existence of an uncertain Bayesian equilibriun will depend accordingly on the existence of an equilibrium in these corresponding static games.

23 We refer the reader to Lehrer and Smorodinsky (1994) for some recent work which has implications for this issue.

24 Nachbar (1995) raises interesting issues related to existence. He shows that in a class of infinitely repeated 2 by 2 games. if the sets of strategies that each player considers as plausible are sufficiently diverse in a specific sense. then it is not possible to have beliefs over the plansible strategies such that players learn and have plausible strategies that best respond to those beliefs. Although. the incompatibility depends on the class of games considered and appropriateness of the diversity condition (which is not easy to gauge). Nachbar's results suggest that there is still much to understand about the beliefs that are compatible with both learning and the existence of an equilibrium. Also. although Nachbar considers repeated games, there may be some analog of his results for recurring games. Such an analog is not obvious, however. since in a recurring game players care only about their own stages, thus substantially weakening the best response requirement. and are learning a distribution over types rather than a specific type. 


\section{References}

Aluanx. R. J. [1992], "Irrationality in Game Theory:" In P. Dasgupta. D. Cale, O. Iart. and E. Maskin. Economir Analysis of Markets and Games: Essays in Honor of Frank Ilahn. MIT Press, C'ambridge MA. pp 21.4227.

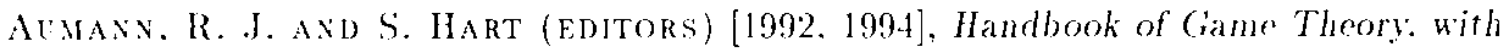
Economic Applications, (Vol. 1, 2). North Holland: Amsterdam.

AcMaxid. R. J. AND M. Maschler [196i]. "Repeated Games with Incomplete Information: A Survey of Recent Resilts." Mathematica, Vol. 116, pp. 287-103.

Battigalli, P., M. Gill, and M.C. Molnari [1992]. "Learning and Convergence to Equilibrium in Repeated Strategic Interactions: An Introductory Survey:" Richerche Economiche. Vol. 96. pp. 3335-378.

BlaCkWELl. D. AND L. Dinase [1962]. "Merging of Opinions with Increasing Information." Annals of Mathematical Statistics. Vol. 38. pp. $882-886$.

Fedfinhers, D. AND D. KREPS [1988]. "A Theory of Learning. Experimentation and Equilibrium in Cames." mimeo: Stanford University.

Fl'dexherci. D. Axd D. Kreps [1991]. "Learning in Extensive Form Games. I: Self ('onfirming Equilibria." Games and Economic Behavior. Vol. S. pp. 20-55.

Fidexherci, D. and D. Levine [1993a]. "Self ('onfirming Equilibrium." Econometrica. Vol. 61. pp. 52:3 5.15.

Fenexher(i. D) axd D. Levixe [1993b], "Steady State Learning and Sash Equilibrium." Econometrica. Vol. 61, pp. 547-5i3.

Fldexherci, D. and J. Tirole [1991]. "Perfect Bayesian Equilibrium and Sequential Equilibrium." Journal of Economir Theory: Vol. 53. pp. 236 260.

HARSAXYt, J.C. [1967-1968], "Games of Incomplete Information Played by Bayesian Players. Parts I III." Managment Science. Vol. 14. pp. 159-182. 320-331. 1\$6 502.

JACKSOX. M. AND E. KALA [1995a]. "Recurring Bullies, Trembling (hallengers, and Learning." mimro.

Jacksox, M. axo E. Kala [1995b]. "Learning to Play Perfectly in Recurring Extensive Form Cames," mimeo.

Jokdx. J. [1991], "Bayesian Leaming in Normal form Ciames," (iames and Economic Beharior. Vol. 3. pp. 60-81.

Jorbax. J. [1992]. "The Exponential ('onvergence of Bayesian Leaming in Nomal form (iames." (Games and Economic Behavior. Vol. . pp. 202-217.

Jordax. J. [1993]. "Three Problems in Learning Mixed-Strategy Equilibria," Cames and Fronomic Behavior. Vol. 5. pp. 368 386.

Kalai, E. and E. Lenrer [1993a], "Rational Learning Leads to Nash Equilibrium," Econometrica. Vol. 61. pp. 1019-1045. 
Kalai. E. Axi E. Lehrer [1993b]. "Subjective Equilibrium in Repeated Cames," Econo-

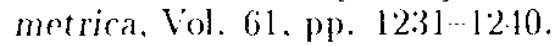

KREPS. D.. P. MILGROM. D.J. ROBERTS. ANI) R. Wilsox [1982]. "Rational (ooperation in the Finitely Reprated Prisoners' Dilemma," Journal of Economic Theory. Vol. 27. pp. 2.45252.

Lehrer. E. ANn R. SMorodissy [199.1]. "Repeated Large (ames with Incomplete Information." mimeo Tel-Aviv Iniversity.

Myersox. R. [1991]. (iame Theory: Analysis of Conflict. Harvard Cuiversity Press. ('ambridge NA.

NACHBAR, J. [1995]. "Prediction, Optimization, and Rational Learning in Cames," mimeo: Washington Iniversity in St. Louis.

NAsh. J. [1950], "Non-Cooperative Ciames," PhD dissertation. Princeton Cniversity Drpartment of Mathematics.

RadNer. R. [1980], "Collusive Behavior in Noncooperative Epsilon Equilibrium of Oligopolies with Long but Finite lives," Journal of Economic Theory. Vol. 22. pp. 13615.

Rrbinstes, A. AND A. Woldseky [1990], "Rationalizable ('onjectural Equilibrium: Betwen Nash and Rationalizability." forthcoming: Cames and Economic Beharior.

Seltex. R. [1975], "Reexamination of the Perfectness concept for Equilibrium Points in Extensive (Games." International Jonnal of (ame Theors. Vol. 4. pp. 25-55).

Shapley. L.S. [195:3]. "Stochastic Cames." Proceedings of the National Academy of Science L.S.A. Vol. 39. pp. 325 332.

Wa Dame. E. [1987]. Stability and Prefection of Nash Equilbria. Springer Verlag.

Yolve. P. [1993]. "The Evolution of conventions." Econometrica. Wol. 61. Pp. 3T R.t. 